\title{
Which assessments are used to analyze neuromuscular control by electromyography after an anterior cruciate ligament injury to determine readiness to return to sports? A systematic review
}

Angela Blasimann ${ }^{1,2^{*}}$, Irene Koenig ${ }^{1}$, Isabel Baert ${ }^{2}$, Heiner Baur $^{1}$ and Dirk Vissers ${ }^{2}$

\begin{abstract}
Background: Adequate neuromuscular control of the knee could be one element to prevent secondary injuries after an anterior cruciate ligament (ACL) injury. To assess neuromuscular control in terms of time, amplitude and activity, electromyography (EMG) is used. However, it is unclear which assessments using EMG could be used for a safe return to sports (RTS). Therefore, we aimed to summarize EMG-related assessments for neuromuscular control of the knee in adult patients after an ACL injury to decide upon readiness for RTS.

Methods: This systematic review followed guidelines of Preferred Reporting of Items for Systematic Reviews and Meta-Analyses (PRISMA) and Cochrane recommendations. MEDLINE/PubMed, EMBASE, CINAHL, Cochrane Library, Physiotherapy Evidence Database (PEDro), SPORTDiscus and the Web of Science were searched from inception to March 2019 and updated in November 2020. Studies identifying electromyographic assessments for neuromuscular control during dynamic tasks in adult, physically active patients with an anterior cruciate ligament injury were eligible and qualitatively synthesized. Two independent reviewers used a modified Downs and Black checklist to assess risk of bias of included studies.
\end{abstract}

Results: From initially 1388 hits, 38 mainly cross-sectional, case-controlled studies were included for qualitative analysis. Most studies provided EMG outcomes of thigh muscles during jumping, running or squatting. Outcomes measures described neuromuscular control of the knee in domains of time, amplitude or activity. Risk of bias was medium to high due to an unclear description of participants and prior interventions, confounding factors and incompletely reported results.

Conclusions: Despite a wide range of EMG outcome measures for neuromuscular control, none was used to decide upon return to sports in these patients. Additional studies are needed to define readiness towards RTS by assessing neuromuscular control in adult ACL patients with EMG. Further research should aim at finding reliable and valid, EMG-related variables to be used as diagnostic tool for neuromuscular control. Moreover, future studies should aim

*Correspondence: angela.blasimann@bfh.ch

1 Department of Health Professions, Division of Physiotherapy, Bern University of Applied Sciences, Murtenstrasse 10, 3008 Bern, Switzerland

Full list of author information is available at the end of the article

(c) The Author(s) 2021. Open Access This article is licensed under a Creative Commons Attribution 4.0 International License, which permits use, sharing, adaptation, distribution and reproduction in any medium or format, as long as you give appropriate credit to the original author(s) and the source, provide a link to the Creative Commons licence, and indicate if changes were made. The images or other third party material in this article are included in the article's Creative Commons licence, unless indicated otherwise in a credit line to the material. If material is not included in the article's Creative Commons licence and your intended use is not permitted by statutory regulation or exceeds the permitted use, you will need to obtain permission directly from the copyright holder. To view a copy of this licence, visit http://creativecommons.org/licenses/by/4.0/. The Creative Commons Public Domain Dedication waiver (http://creativeco mmons.org/publicdomain/zero/1.0/) applies to the data made available in this article, unless otherwise stated in a credit line to the data. 
at more homogenous groups including adequately matched healthy subjects, evaluate gender separately and use sport-specific tasks.

Registration The protocol for this systematic review was indexed beforehand in the International Prospective Register of Systematic Reviews (PROSPERO) and registered as CRD42019122188.

Keywords: Knee, Anterior cruciate ligament injuries, ACL, Electromyography, EMG, Rehabilitation, Patient outcome assessment, Neuromuscular control, Return to sports, RTS

\section{Background}

Anterior cruciate ligament (ACL) injuries happen quite frequently and concern athletes (0.15 injuries per 1000 athletic exposures (AEs)) but also the active part of the general population $[1,2]$. Most ACL injuries are due to a non-contact, multiplane mechanism [3] and may lead to instability, secondary meniscal injury or even knee osteoarthritis in the long run [4]. Consequently, this injury means several months or even years of physical impairment with wide consequences for the patients concerning return to work, return to activity or return to sport (RTS). RTS rates between 63 and $97 \%$ are reported for patients after ACLR [5, 6]. Most elite athletes return to sports return earlier than non-elite athletes [5], on average within 12 months [6]. However, it remains unclear whether this approach is safe [6], omitting further injury, respectively. Athletes after ACLR returning to highdemanding sports (including jumping, pivoting and hard cutting) show a more than fourfold increase in reinjury rates over two years [7]. More than 5\% of athletes with an ACLR sustain a re-rupture of the graft $[6,8]$ in the ipsilateral knee after RTS. The risk for an ACL tear in the contralateral knee is as double as high (11.8\%) even five years or longer after an ACLR [8]. Overall, the recurrence rates even after successful ACLR and subsequent rehabilitation are high $(29.5 \%$ or $1.82 / 1000 \mathrm{AEs})$, with a tear of the ACL graft (9.0\%), an ACL injury of the opposite leg (20.5\%), muscle injuries on the ipsilateral side or even bilateral consequences $[9,10]$.

It is known that ipsilateral deficits in clinical knee function and knee laxity persist even years after ACLR [11, 12]. ACL patients show altered kinematics and kinetics [13] and different neuromuscular strategies during walking [14], not only in the injured limb but also in the non-affected side $[13,15]$. These changes are referred to neuromuscular adaptations due to altered sensorimotor control [16] and are caused by altered afferent inputs to the central nervous system due to the loss of the mechanoreceptors of the native (original) ACL [17]. Current literature regarding in ACL patients emphasises the importance of understanding consequences of ACL injury regarding neuromuscular control and kinematics [18-20]. To describe neuromuscular control in terms of simultaneously activated agonist/antagonist muscle pairs, generalized knee muscle co-contraction parameters are used [21, 22].

In daily clinical practice, physical performance tests batteries including jumps and tests of muscle function [23] are often used to assess neuromuscular control for RTS. However, there is only limited evidence that passing RTS test batteries-interpreted as having achieved adequate levels of mobility, stability, strength, balance, and neuromuscular control for RTS-reduces the risk for a second ACL injury [24]. Moreover, it remains unclear which measures should be used to bring athletes safely back to RTS with a low risk of re-injury [25]. In conclusion, the currently suggested RTS criteria do not seem to be adequate to assess neuromuscular control of the knee joint to judge upon a safe RTS or even competition. Therefore, meaningful, reliable, valid and accurate diagnostic tools for patients with an ACL injury (either treated surgically or conservatively) are needed and may aid clinical decision-making towards a safe RTS following ACLR. Objective measurements of neuromuscular control should include electromyography (EMG) of involved muscles to judge upon quantity, quality and timing of voluntary activation and reflex activity $[13,20,26]$. However, up to date it is unclear which EMG-related measurements for neuromuscular control are used in patients with an ACL injury to decide upon a safe RTS.

\section{Objectives}

The first objective of this systematic review was therefore to summarize the scientific literature regarding EMGrelated assessments for neuromuscular control in adult, physically active patients with an ACL injury (either treated surgically or conservatively) during functional tasks. The second aim was to analyze whether these assessments for neuromuscular control were used to decide upon readiness for RTS in these patients.

\section{Methods}

This systematic review was planned, conducted and analyzed according to the guidelines of Preferred Reporting of Items for Systematic Reviews and Meta-Analyses (PRISMA) [27] and followed the recommendations of Cochrane group [28]. 
The protocol for this systematic review was indexed beforehand in the International Prospective Register of Systematic Reviews (PROSPERO) and got the registration number CRD42019122188.

\section{Eligibility criteria}

To define the relevant key words for the literature search, the Participants-Intervention-Control-Outcome-Study design (PICOS) scheme was used as follows (Table 1).

Studies were considered eligible for this systematic review if they met the following inclusion criteria: Study participants-either females, males or both-had to be 18 years or older, suffer from an ACL injury-either treated conservatively or surgically-with a time since injury/surgery of six months at least, be athletes or physically active people who participate in sports activities on a regular basis (as defined by each study, e.g. Tegner Activity Score (TAS) $\geq 3$ ) to get data to decide upon RTS. Moreover, included studies had to have used active or functional tasks such as walking, stair climbing or jumps, applied assessments for neuromuscular control of lower limb muscles using EMG, be original articles published in peer-reviewed, scientific journals in English, German, French, Italian or Dutch, and available as full texts. Exclusion criteria were model-driven approaches, animals or cadavers, comparisons of surgical techniques, passive or non-functional tasks (such as isokinetic measurements for strength and isometric muscle activity), editorials, conference abstracts, book chapters, theses, systematic reviews and meta-analyses.

\section{Data sources}

The search was effectuated from inception until March 2019 and updated in November 27th, 2020 in the electronic databases MEDLINE/PubMed, EMBASE, CINAHL, Cochrane Library, Physiotherapy Evidence Database (PEDro), SPORTDiscus and in the Web of Science. To ensure new articles matching the search terms, e-mail alerts were established from each of the databases if possible [29]. Furthermore, a hand search was done using the reference lists of included articles to identify additional and potentially eligible articles that had been missed in the electronic database searches. The hits from these two additional sources were also screened for eligibility applying the same criteria as for the articles from the database search.

\section{Search strategy}

In all sources, the advanced search mode was used if available. A search matrix combining relevant keywords (if possible $\mathrm{MeSH}$-terms) with the Boolean operators AND and OR was used and customized for searches in all databases if necessary (see Additional file "Search string for MEDLINE/PubMed" 1): "anterior cruciate ligament/anterior cranial cruciate ligament/ACL"; "anterior cruciate ligament injuries/strains and sprains/rupture/ tear/injury/deficiency"; "anterior ligament reconstruction/anterior cruciate ligament/surgery/reconstructive surgical procedures/orthopedic procedure/ orthopedic procedure/tendon graft/tendon transfer/conservative treatment/non-surgical/rehabilitation/physical therapy modalities/physiotherapy/kinesiotherapy/ exercise/instruction/resistance training/neuromuscular training/postoperative care"; "neuromuscular control/ neuromuscular activity/sensorimotor control/muscle activity/active stability"; "electromyography/EMG/electromyogram/amplitude/timing/mean activity/peak activity/duration of activity/onset/offset/on-off-pattern/ pre-activity/latency/reflex response". In the updated search, articles were filtered by date of publication, with the aim of including only those published between March 2019 and November 2020.

\section{Study selection}

All hits obtained by the database searches were downloaded to the Rayyan reference management platform [30] and inserted into EndNote (Clarivate Analytics, Philadelphia, USA). Prior to screening, duplicates were

Table 1 Overview of PICOS criteria for key word definitions

\begin{tabular}{|c|c|}
\hline Parameter & Criteria \\
\hline Participants (P) & Adult people (age of 18-65 years) who sustained an ACL injury, either treated conservatively or surgically (repaired with an autograft) \\
\hline Intervention (l) & $\begin{array}{l}\text { Assessment of neuromuscular control, active knee stability, sensorimotor control, active stability of the lower limb or similar during } \\
\text { dynamic activities }\end{array}$ \\
\hline Control (C) & Uninjured limb/contralateral side or contralateral lower limb of the ACL-injured participant, or a healthy control group \\
\hline Outcomes $(\mathrm{O})$ & $\begin{array}{l}\text { Any EMG-related outcome describing neuromuscular activity/control in domains of time, amplitude etc.; parameters describing EMG } \\
\text { activity of lower limb muscles; related to EMG variables, such as amplitude, timing, mean or peak activity, duration of activity, onset } \\
\text { and offset/on-off-pattern respectively, pre-activity, latency, reflex response }[14,20]\end{array}$ \\
\hline Study design (S) & $\begin{array}{l}\text { Any laboratory or interventional study, cross-sectional or longitudinal such as randomized controlled trials, clinically controlled trials } \\
\text { without randomization, laboratory/experimental controlled trials etc }\end{array}$ \\
\hline
\end{tabular}


removed. Two authors (AB and $\mathrm{IK}$ ) independently screened title and abstract of the records, one by using the software EndNote (Clarivate Analytics, Philadelphia, USA) and the other with the help of the free software "rayyan" [30]. After screening, full texts of relevant hits were read by the two authors ( $\mathrm{IK}, \mathrm{AB})$ to decide upon inor exclusion. If their decisions did not match, discussion took place until consensus was achieved. If consensus would not have been reached, a third author (IB or HB) would have finally decided upon in- or exclusion of the record in question; however, this was not necessary.

\section{Data collection process and data extraction}

After final decision of all studies, data extraction for each eligible study was performed by the first author (AB) with a predefined Microsoft ${ }^{\circledR}$ Excel (Microsoft Corporation, Redmond WA, USA) spreadsheet as piloted form. The first author $(\mathrm{AB})$ extracted necessary information from each article describing the study design, groups measured and their characteristics, the tasks to be fulfilled by all participants, and all EMG-related assessments or methods used to evaluate neuromuscular control. Furthermore, the chosen assessment for neuromuscular control were judged whether they were used to clear the participants for RTS. The second author (IK) checked the extracted data at random. As all included studies provided enough information to be qualitatively analyzed, it was not necessary to contact corresponding authors for obtaining or confirming data.

\section{Assessment of risk of bias in included studies}

The risk of bias of all the included articles was independently assessed by two raters $(\mathrm{AB}, \mathrm{IK})$ by using the Downs and Black checklist [31] in a modified form [29, 32]. The following categories were evaluated: (1) reporting bias: objectives/hypothesis, main outcomes, patients' characteristics, interventions, principal confounders, main findings, estimates of random variability, actual probability values; (2) external validity bias: study subjects/staff/places/facilities representative; (3) internal validity bias: blinding subjects/assessors, data dredging present, different lengths of follow-up/same time period between intervention and outcome for cases and controls, statistical tests/main outcome measures appropriate; (4) selection bias: patients and controls from same population and over same period of time, randomization, allocation concealed, adjustments for confounding, loss to follow-up; and (5) power analysis (see Additional file "Methodological quality assessment" 2). Each question of the categories was scored with 1 or 2 points if the criterium was fulfilled (answer "yes"), zero points if the answer was "no", "not fulfilled" and an " $X$ " if the criterium was not applicable, e.g. randomization for a case-control or cross-sectional study, "IC" for intrasubject comparison, respectively.

For this systematic review, studies with a total score of 17 or above out of 25 (more than $2 / 3$ of the maximum total score) were considered as being of high methodological quality, showing a "low" risk of bias respectively [29]. Studies which reached 13 to 16 points (more than $50 \%$ of the maximum total score) were rated as being of "medium" quality, and total scores below 13 were rated as being of low methodological quality, "high" risk of bias respectively. As the aim of this systematic review was to summarize the applied measures for neuromuscular control, the methodological quality of the included studies was of secondary interest. Therefore, no study was excluded due to a low total score in the risk of bias assessment.

\section{Results}

Study selection

Hits from the first and the updated database search including e-mail alerts and hand search were screened for duplicates. After applying in- and exclusion criteria according to PRISMA flowchart [27], a total of 38 articles involving 1236 subjects -809 participants with ACLR or ACL deficiency and 427 healthy controls-could be used for qualitative analysis. Reasons for exclusion were participants younger than 18 years, not able to achieve RTS, time since injury or surgery less than six months, static or non-functional task, study design (e.g. systematic review, study protocol), unclear or inadequate outcome, healthy participants or without ACL injury. Included studies had mainly a cross-sectional, case-controlled study design. Details about every step of the search are illustrated in the following flowchart (Fig. 1).

\section{Risk of bias assessment}

Risk of bias of half (19 studies, 50.0\%) of the included studies was medium [13,33-50], six (15.8\%) showed high methodological quality [51-56] and 13 studies (34.2\%) were of low quality [57-69] (Table 2). The main reasons for a medium to low methodological quality were due to an unclear description of participants and prior interventions, confounding factors, and incompletely reported results. Table 2 provides details about the risk of bias assessment for each included study.

\section{Characteristics of included studies \\ Study design}

All included studies were case-control studies, except two which where case series [56] or a single-case study [69]. Two reported a retrospective or secondary data analysis $[52,55]$ or provided a subgroup analysis from a larger trial [45, 47-49, 65-67] (Table 2). Thirty-five 


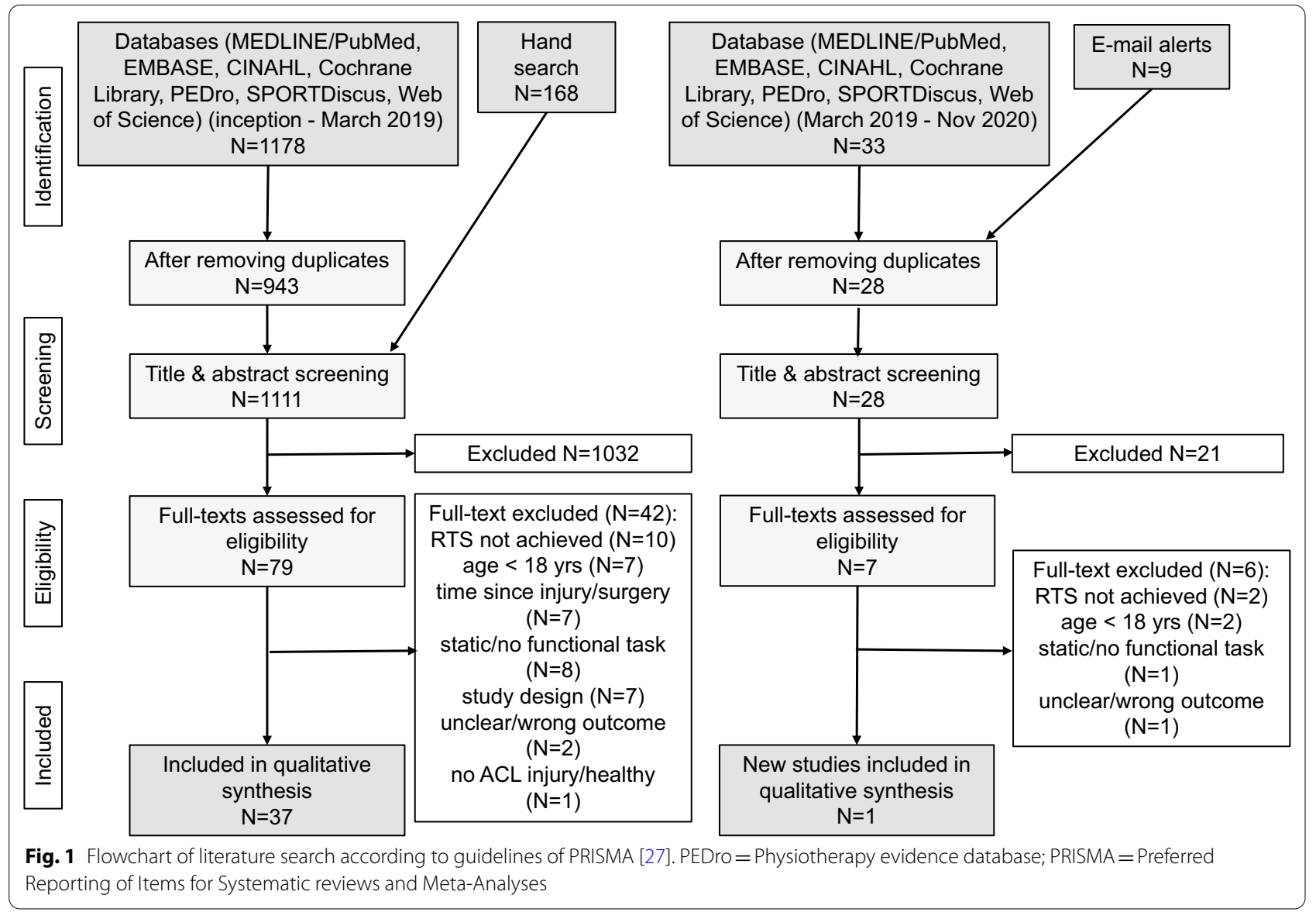

studies compared the ACL participants with at least one control group (other ACL treatment, e.g. surgical versus conservative, or healthy controls), the remaining three studies made a comparison between the injured and the non-injured leg of the participants $[42,44]$ or compared the pre-injury status with follow-up data from pre- and post-surgery [69].

\section{Participants}

The number of included, adult participants with ACL injury varied from $\mathrm{N}=1$ [69] to a maximum of $\mathrm{N}=70$ [62] with a wide range of described physical activity from "normal" [58], "regular" [64], "active in at least one sport" [61], TAS $\geq 3$ [50], minimal $2 \mathrm{~h} /$ week [33, 34] to athletes at level I sports including jumping, pivoting and hard cutting [42, 57, 59], elite soccer players [35, 38, 67, 69] or elite skiers [50]. Some authors restricted study participation to either males [33, 34, 36, 39, 44-46, 50, 58, $60,67]$ or females [50, 51, 64-66, 68, 69], others measured females and males [13, 35, 37, 40, 41, 47-49, 52, $54-57,59,61,62]$. Three studies did not provide any data about the gender of their participants [42, 53, 63]. More patient characteristics of included studies can be found in Table 3.

Details regarding methodological aspects of all included studies are presented in Table 4 below.

\section{Interventions}

The number of muscles assessed ranged from one [37, 45, 61 to ten [59]. Mainly muscle activity of four muscles of the thigh, vastus lateralis, vastus medialis, biceps femoris and semitendinosus, had been assessed. However, there were also studies measuring the adductor longus [39, 62], gluteus medius $[39,65,66]$, gluteus maximus $[41,52,54-$ $56,59,65,66]$, and calf muscles such as soleus, medial and lateral gastrocnemius [47-49, 54-56, 59, 60, 63].

The tasks used were very diverse: there were activities of daily life such as walking on even ground and downhill [33, 47, 53, 57, 58, 62, 63, 68], and stair climbing [13, 49]. Other activities went more towards sports such as running $[44,45,60,67,68]$ and jumping $[36,37,39-42,48$, $50-52,54-56,59,61,65,66,68]$ where mainly the singleleg hop for distance, drop jumps and countermovement jumps were used. Some authors chose typical rehabilitation exercises such as forward lunges [34], Nordic 


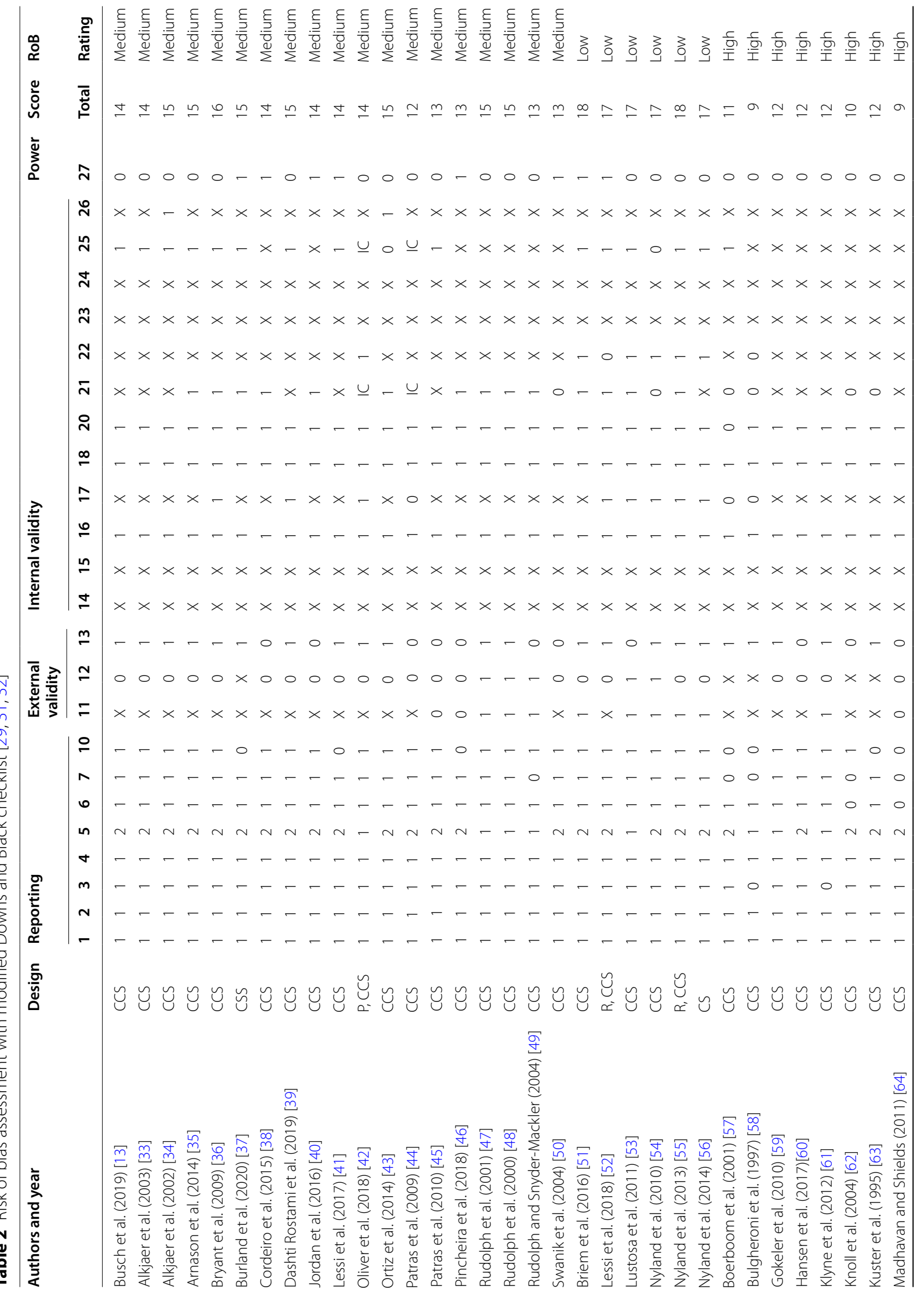




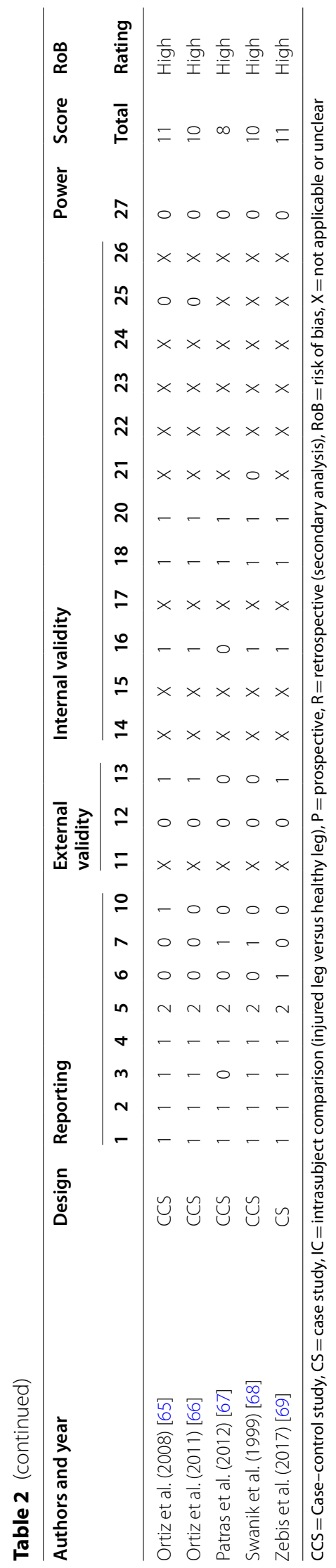




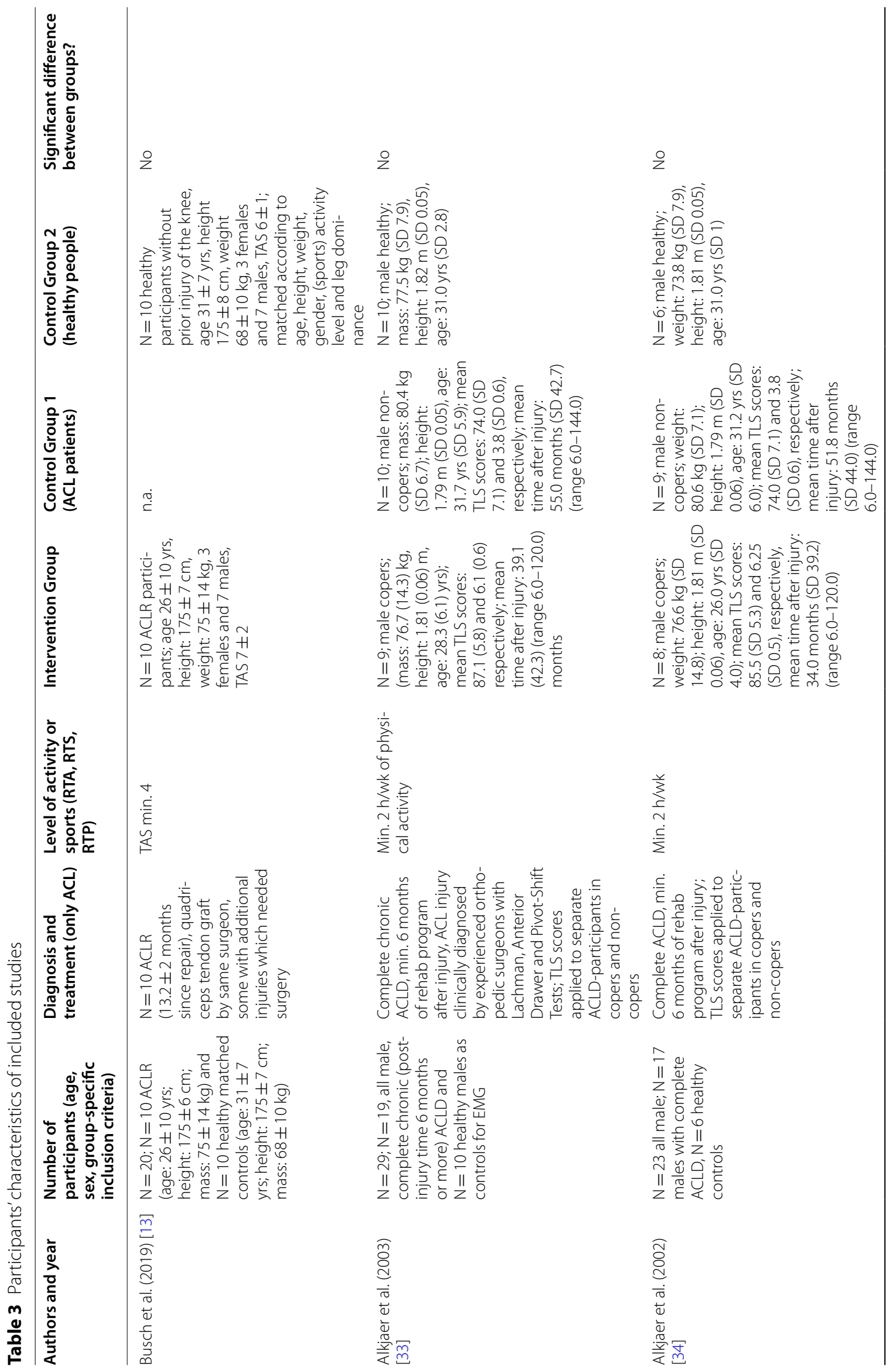




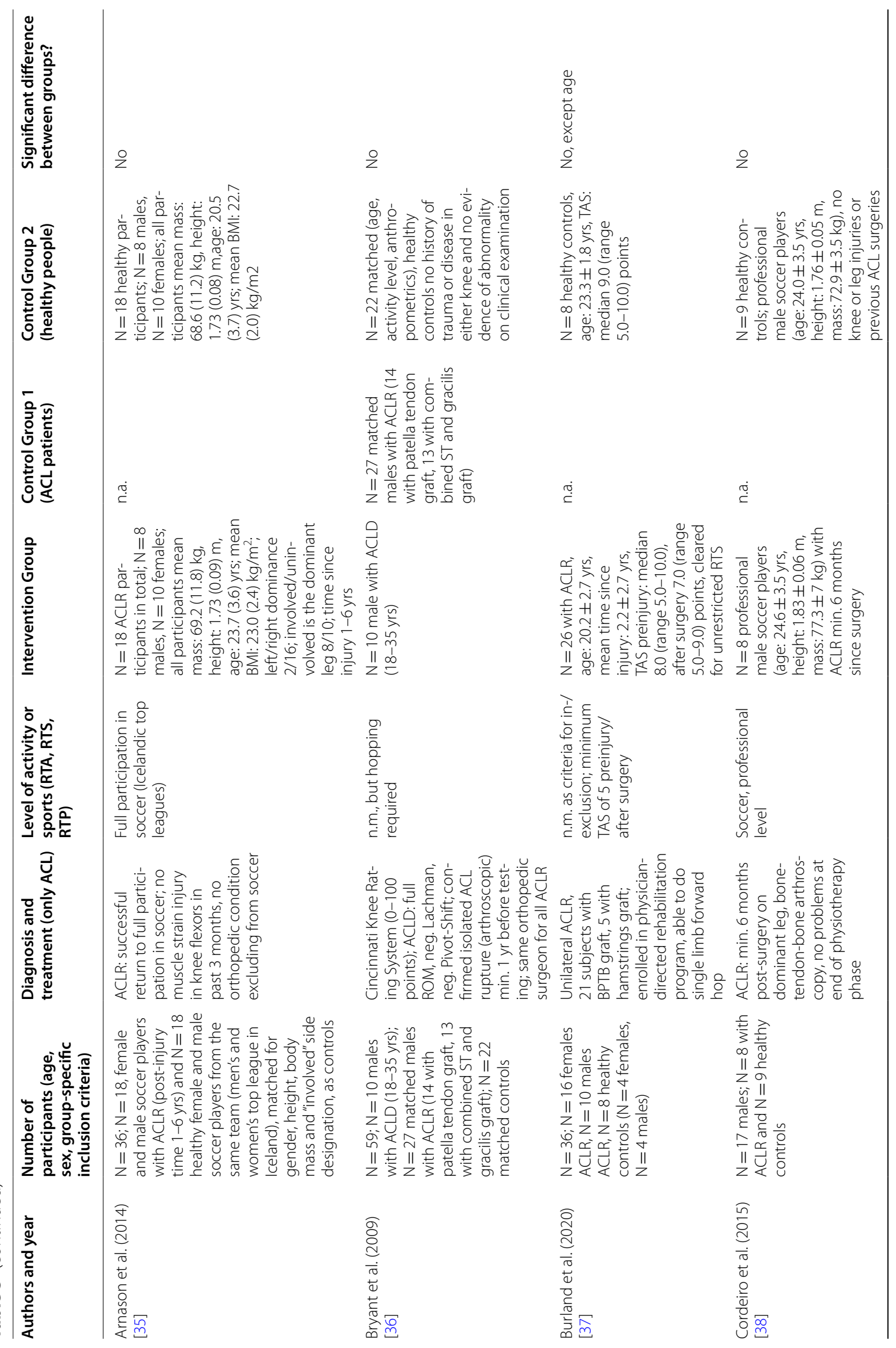




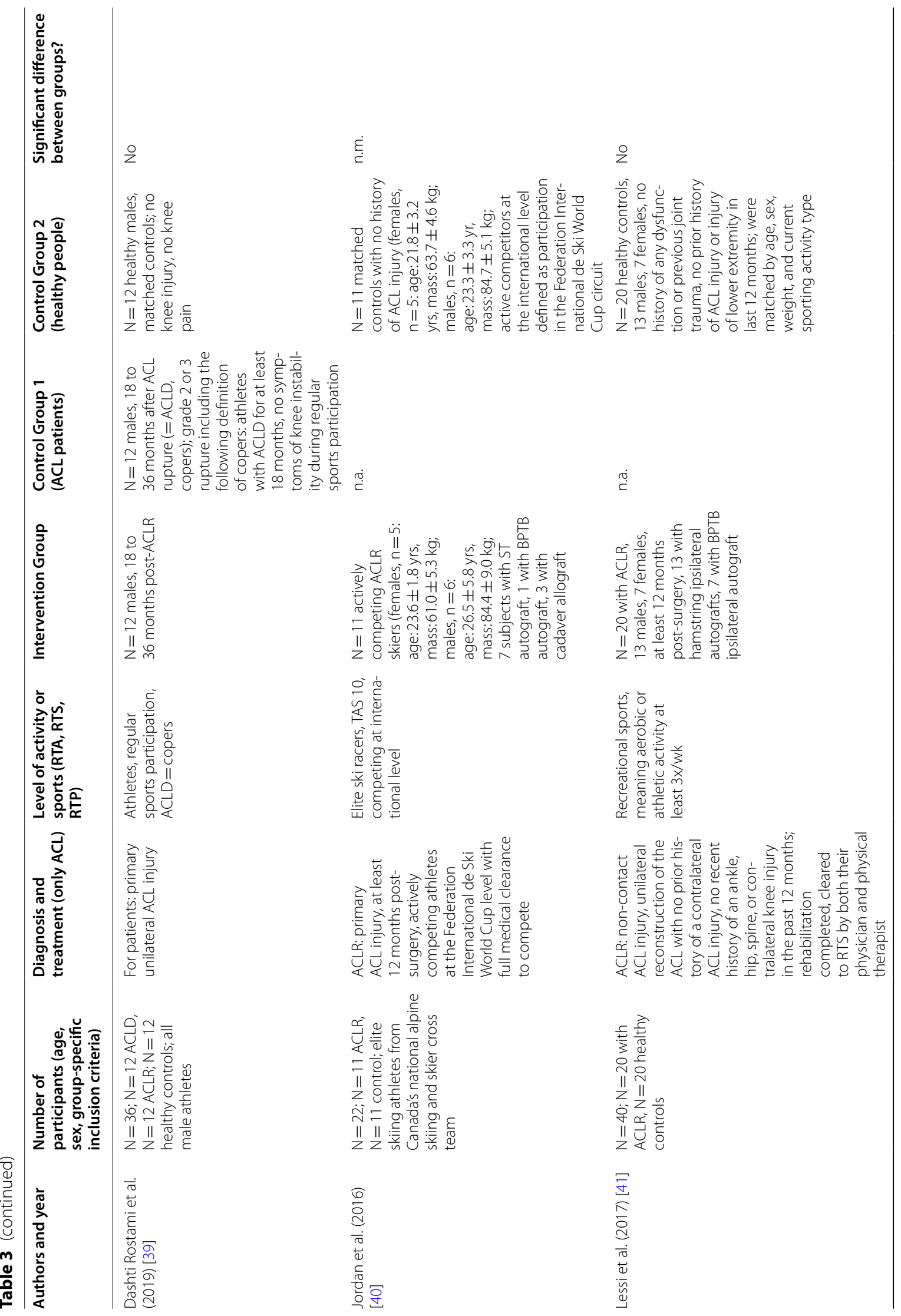




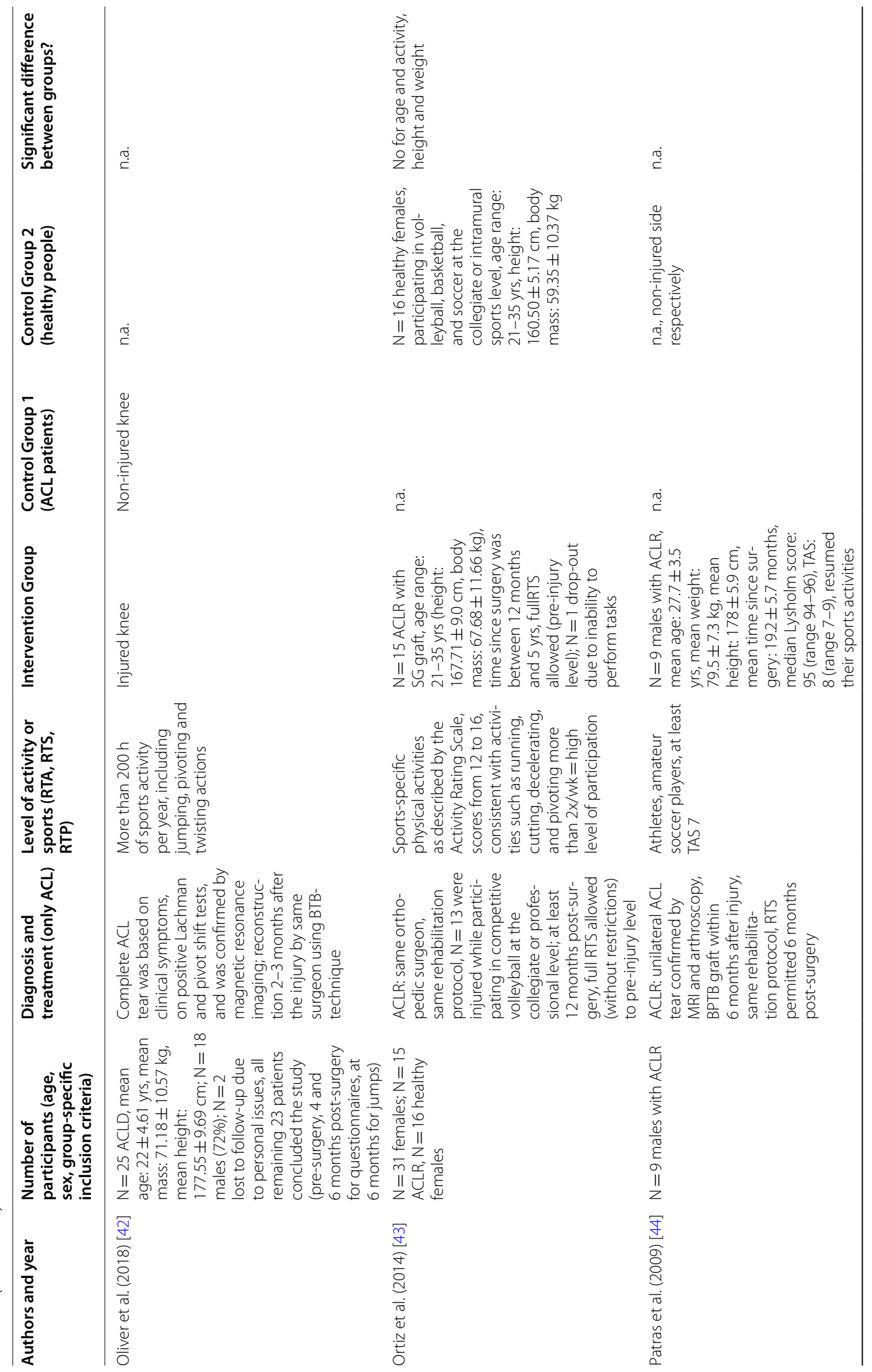




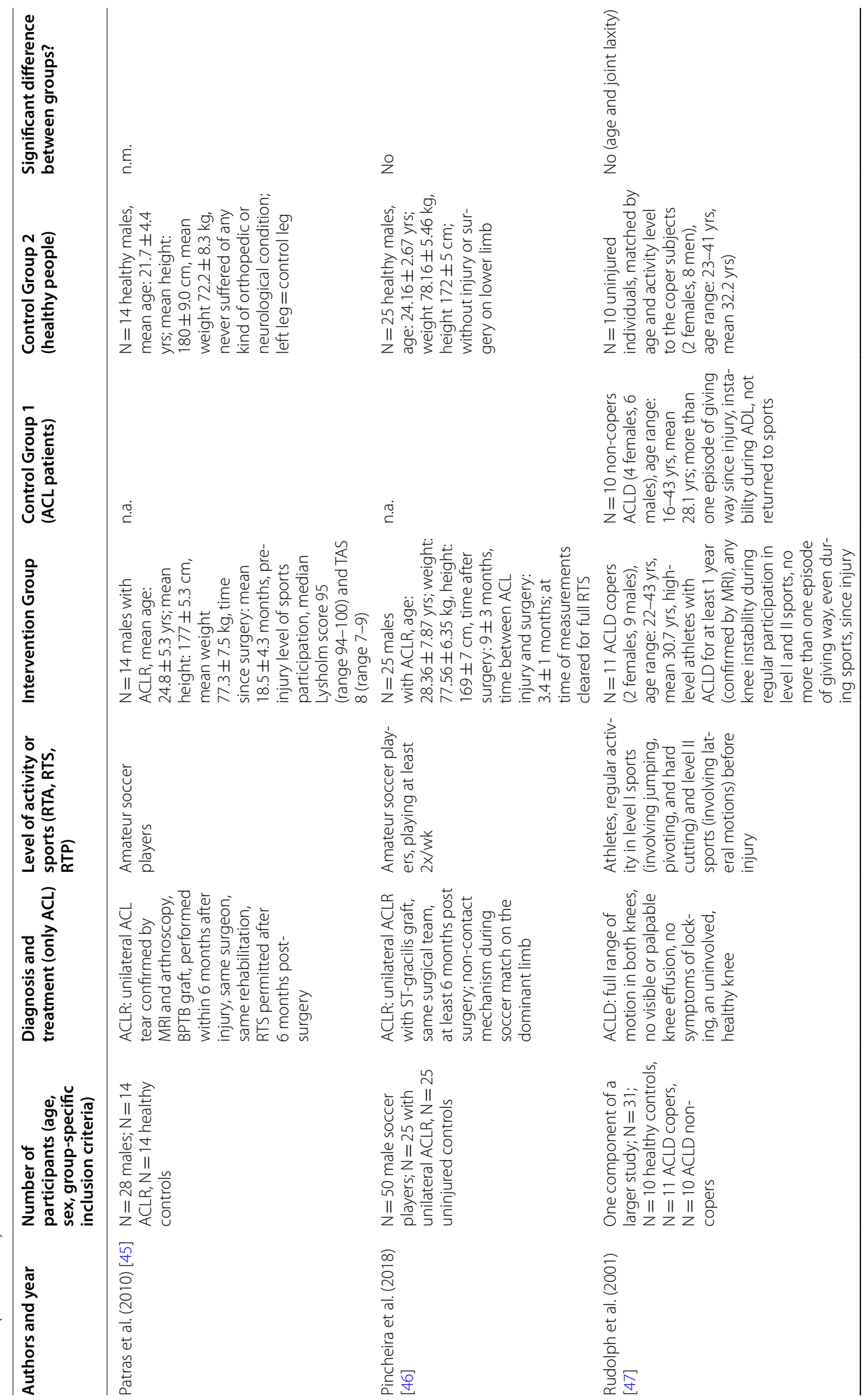




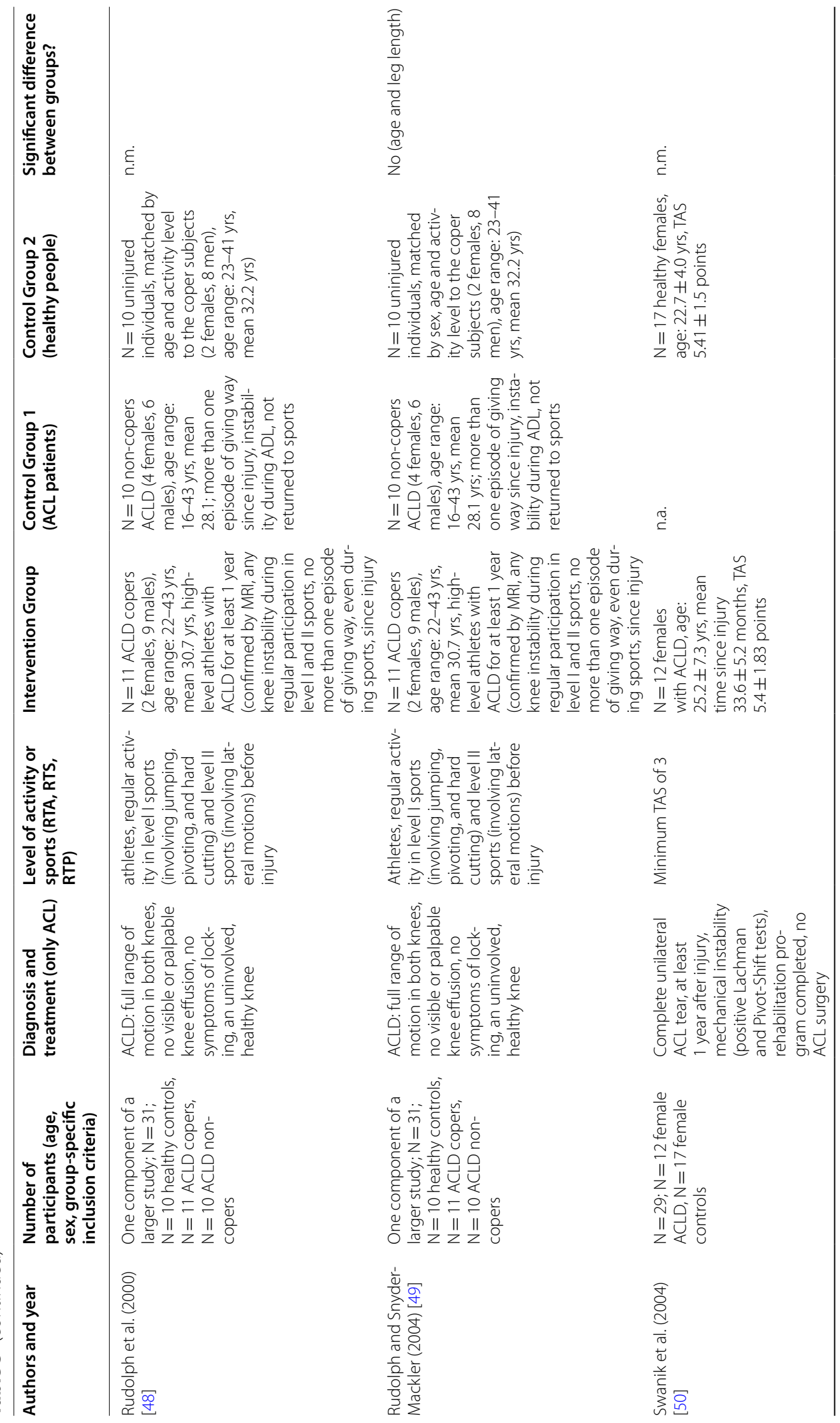




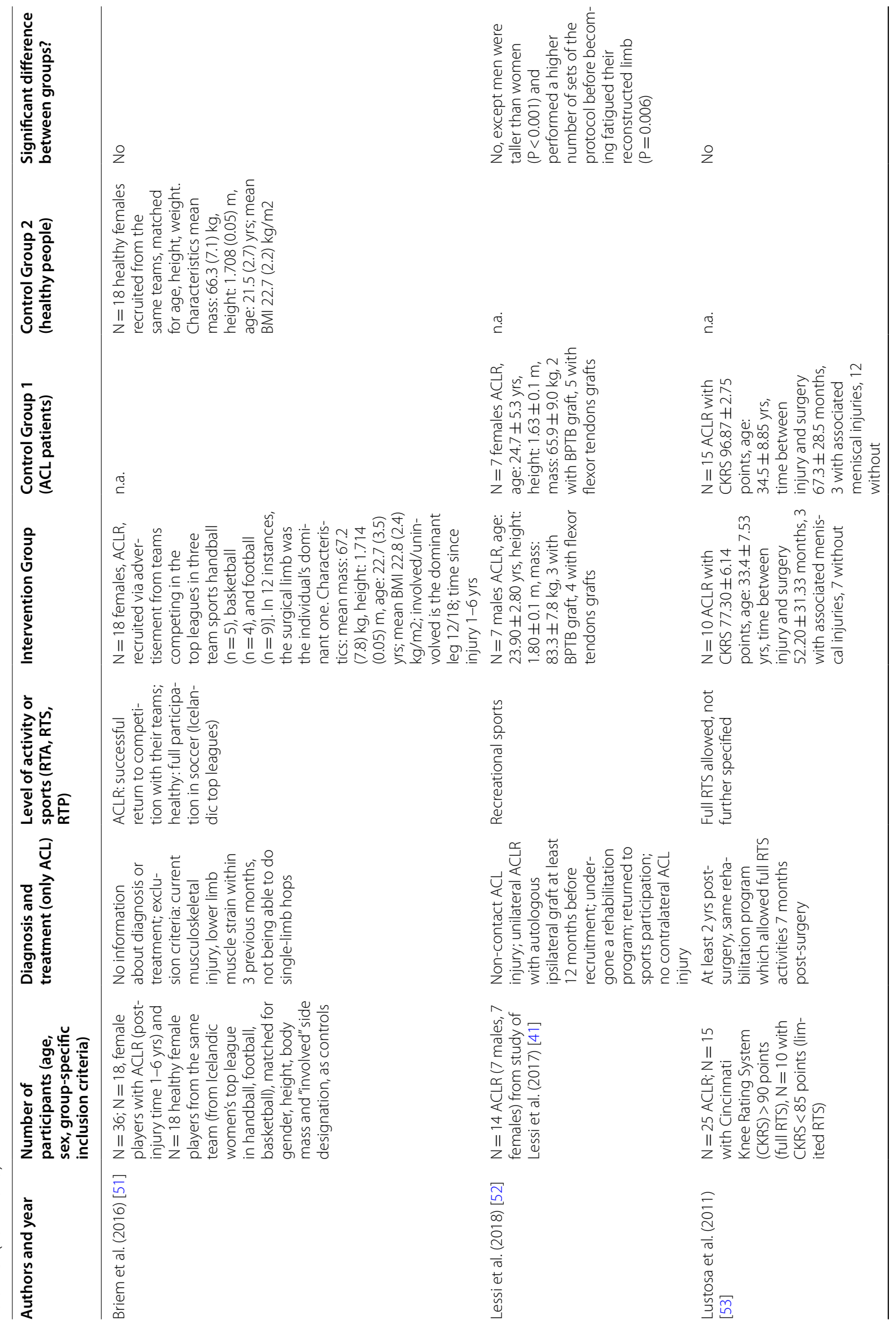




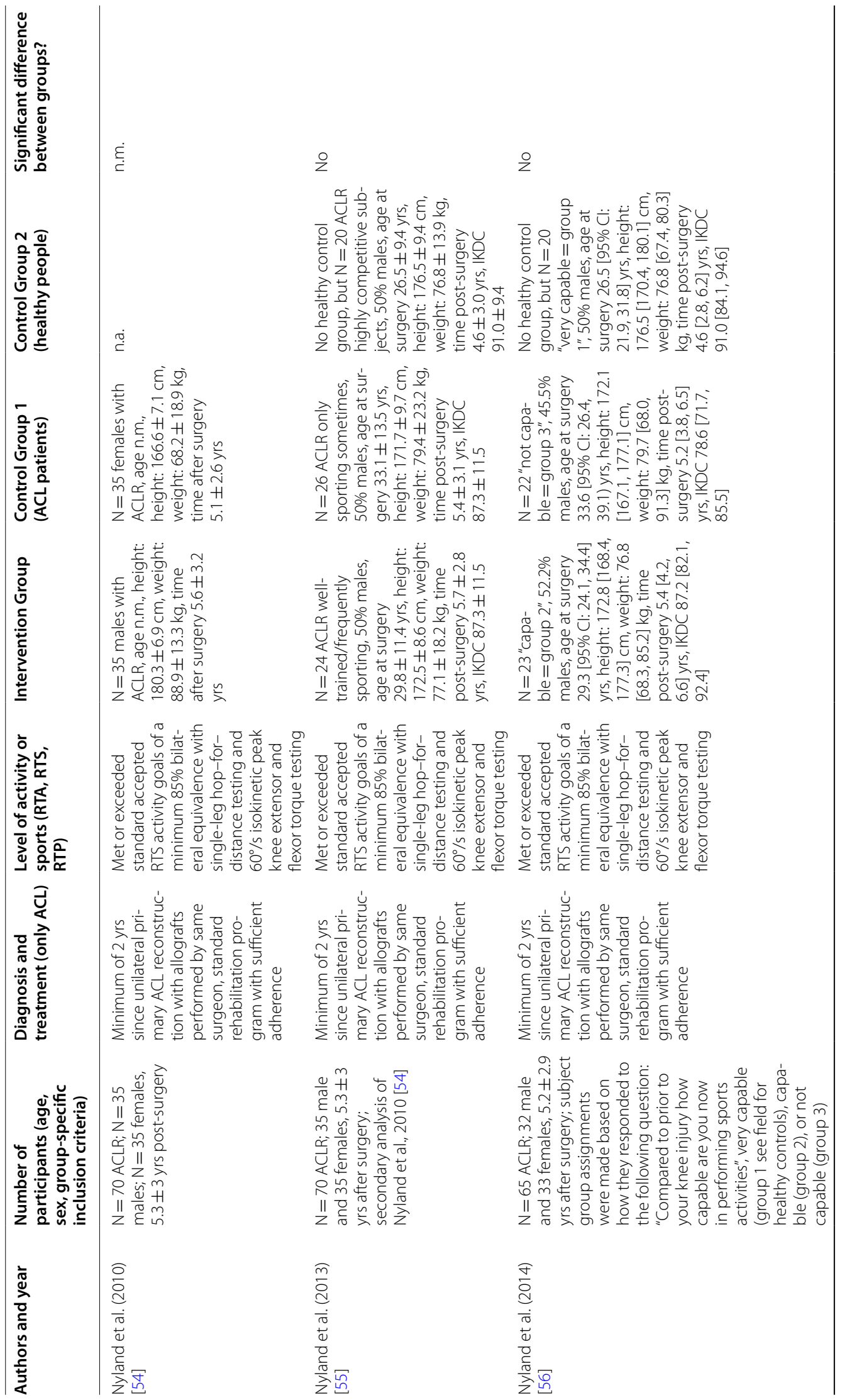




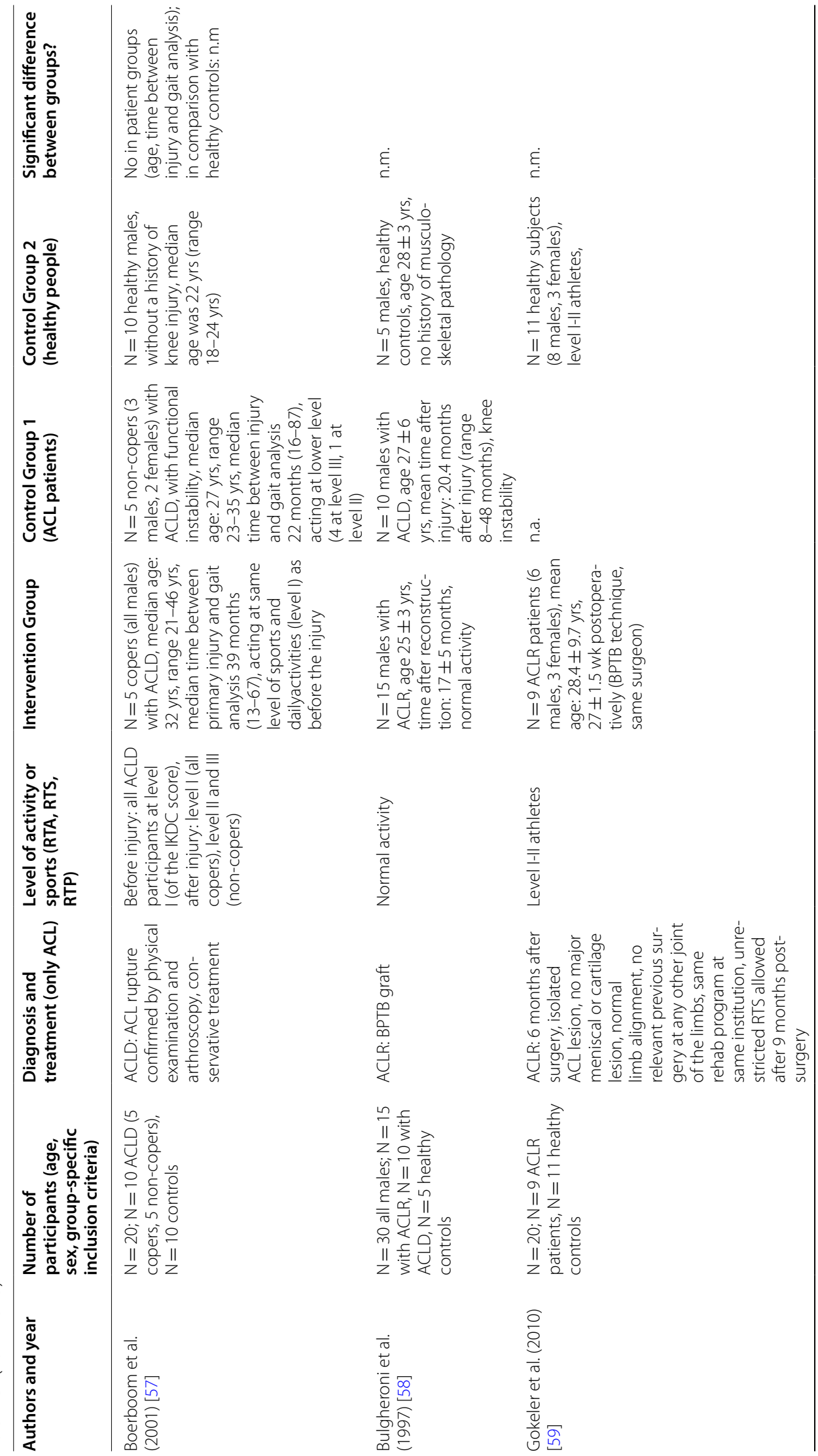




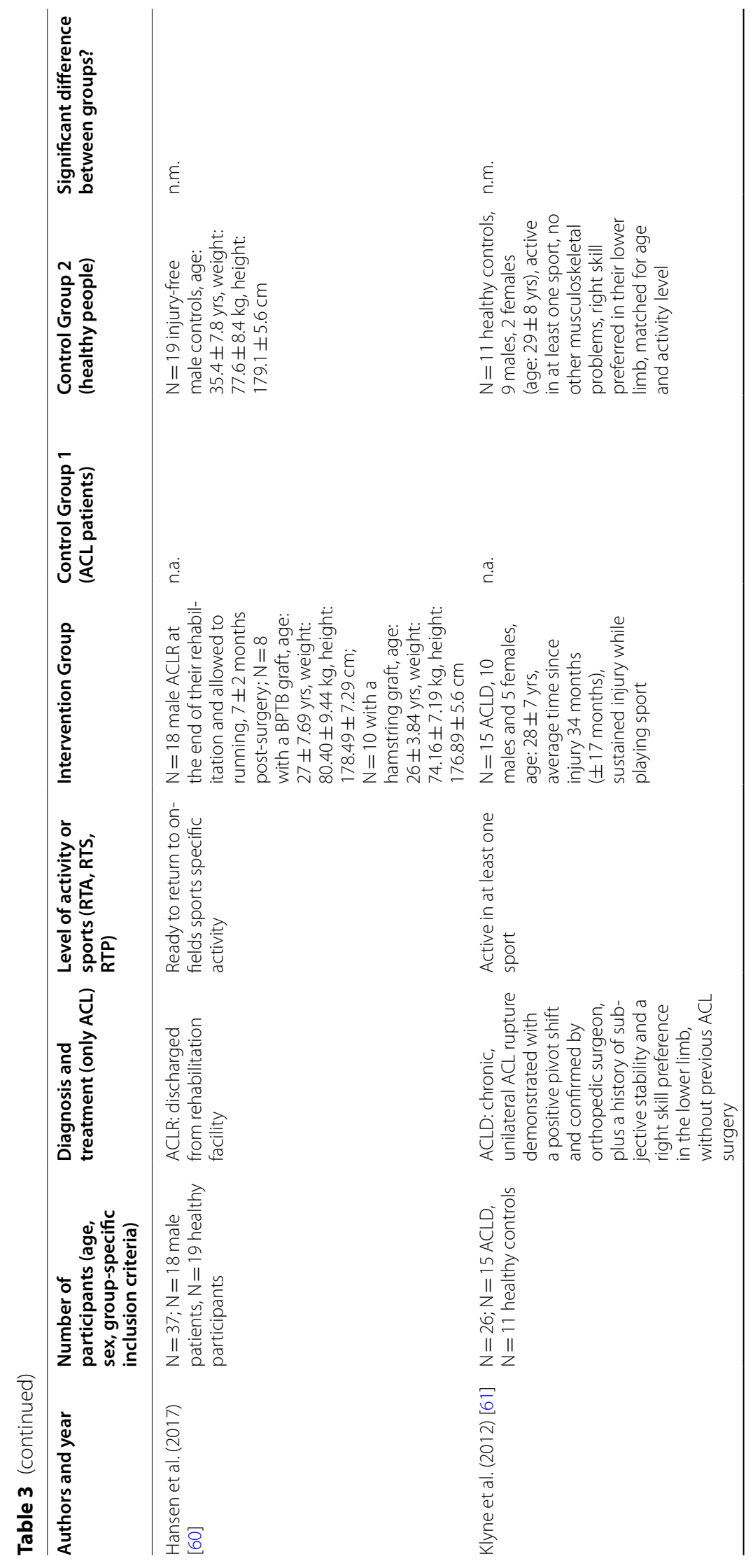




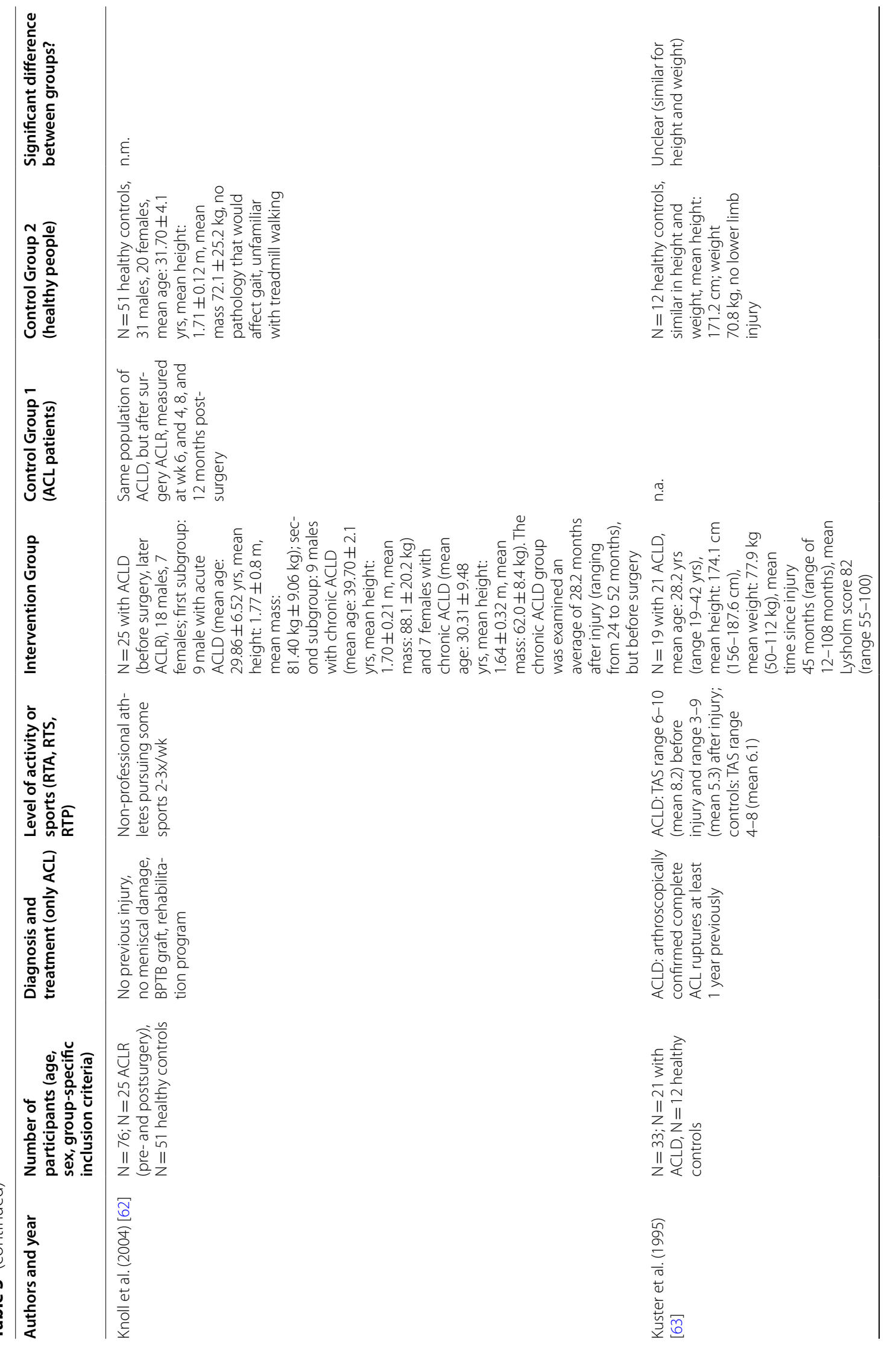




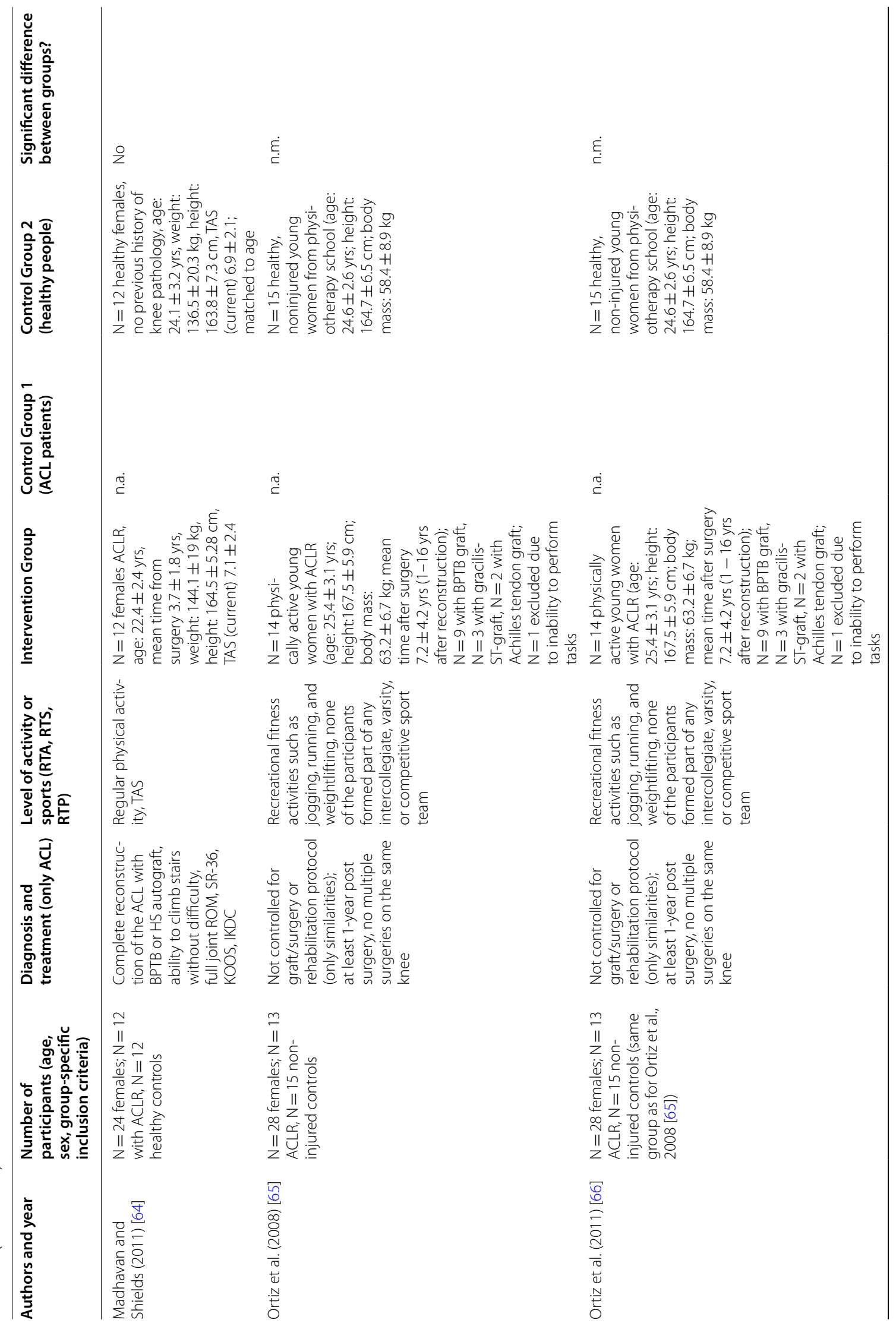




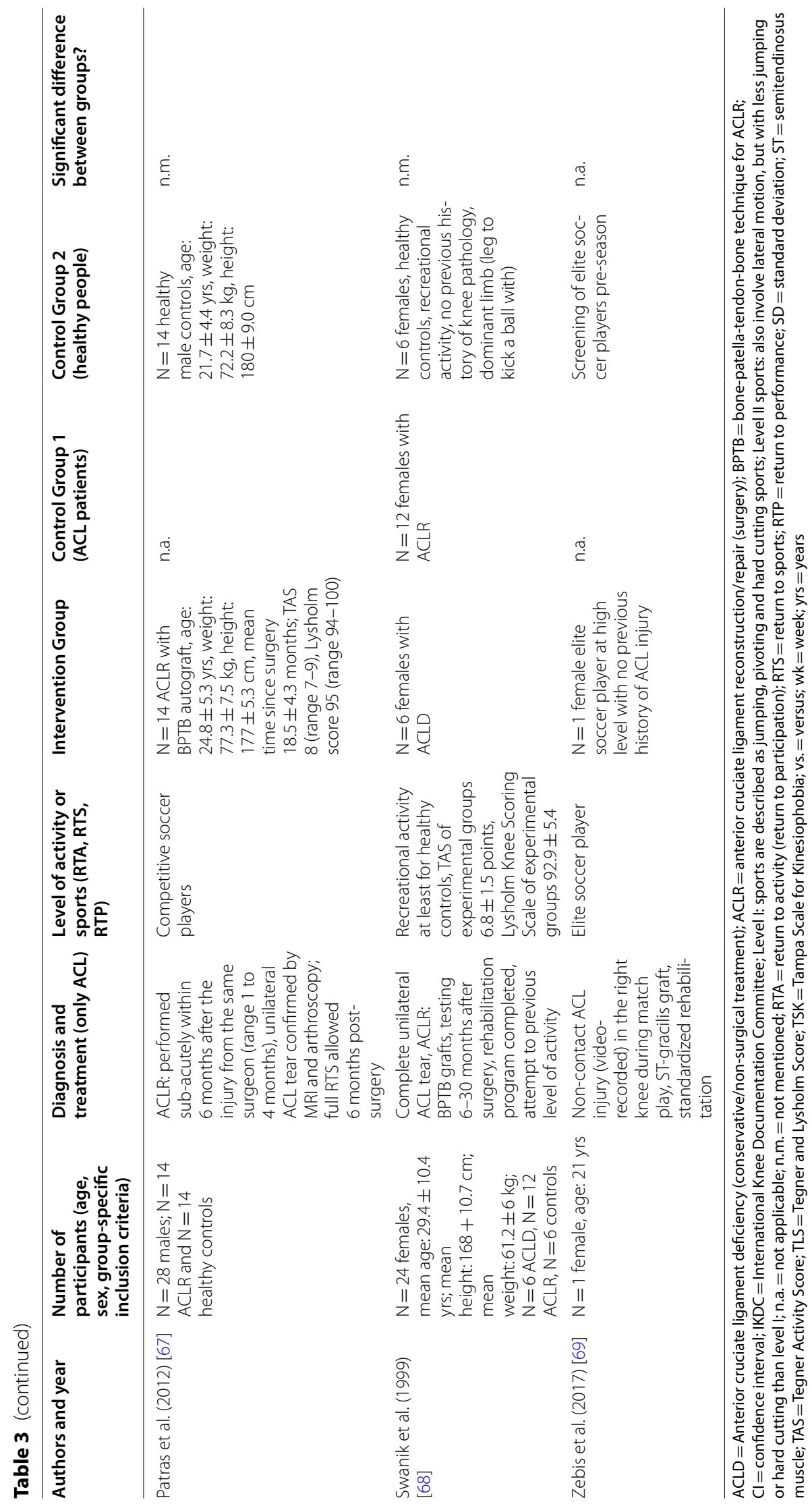




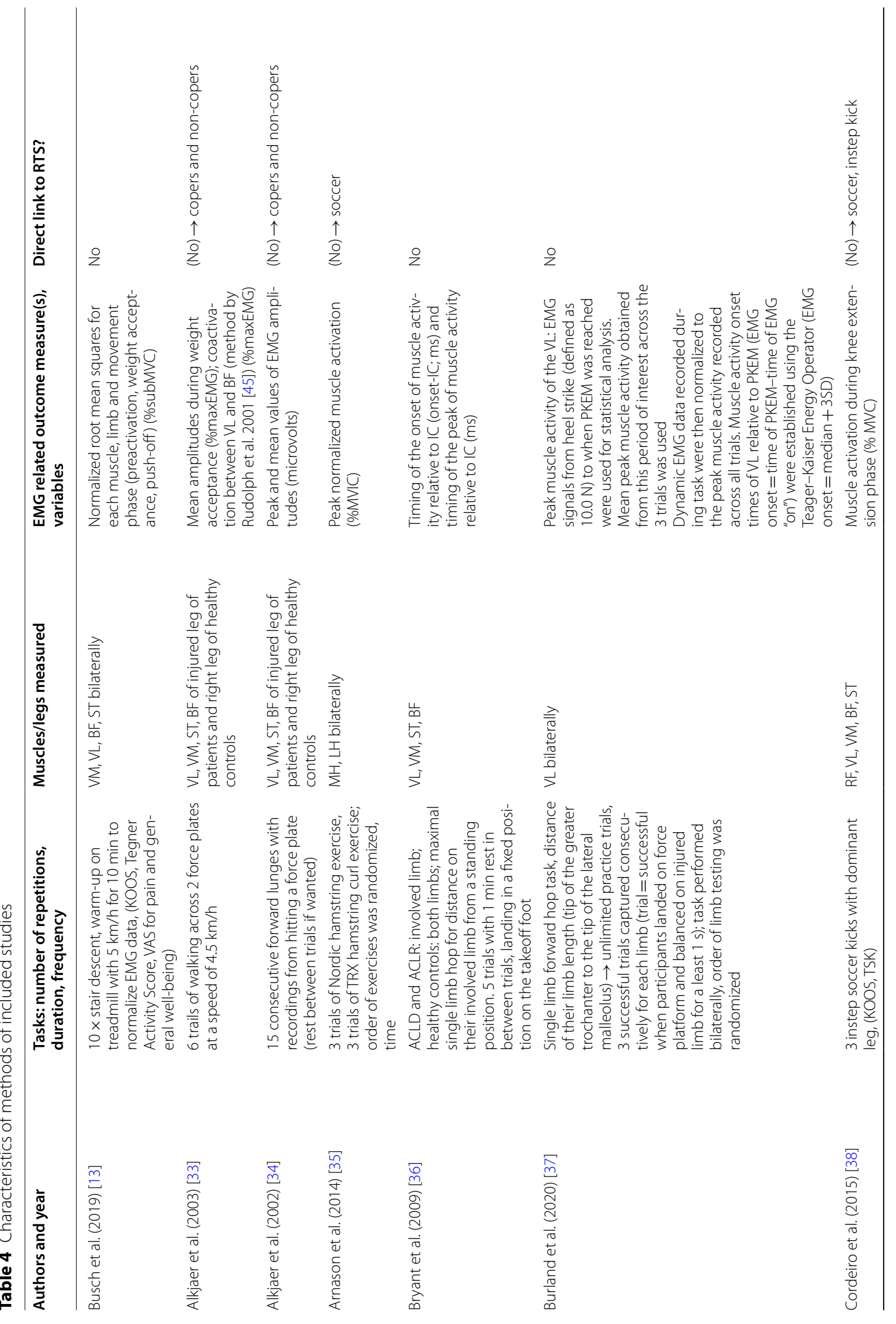




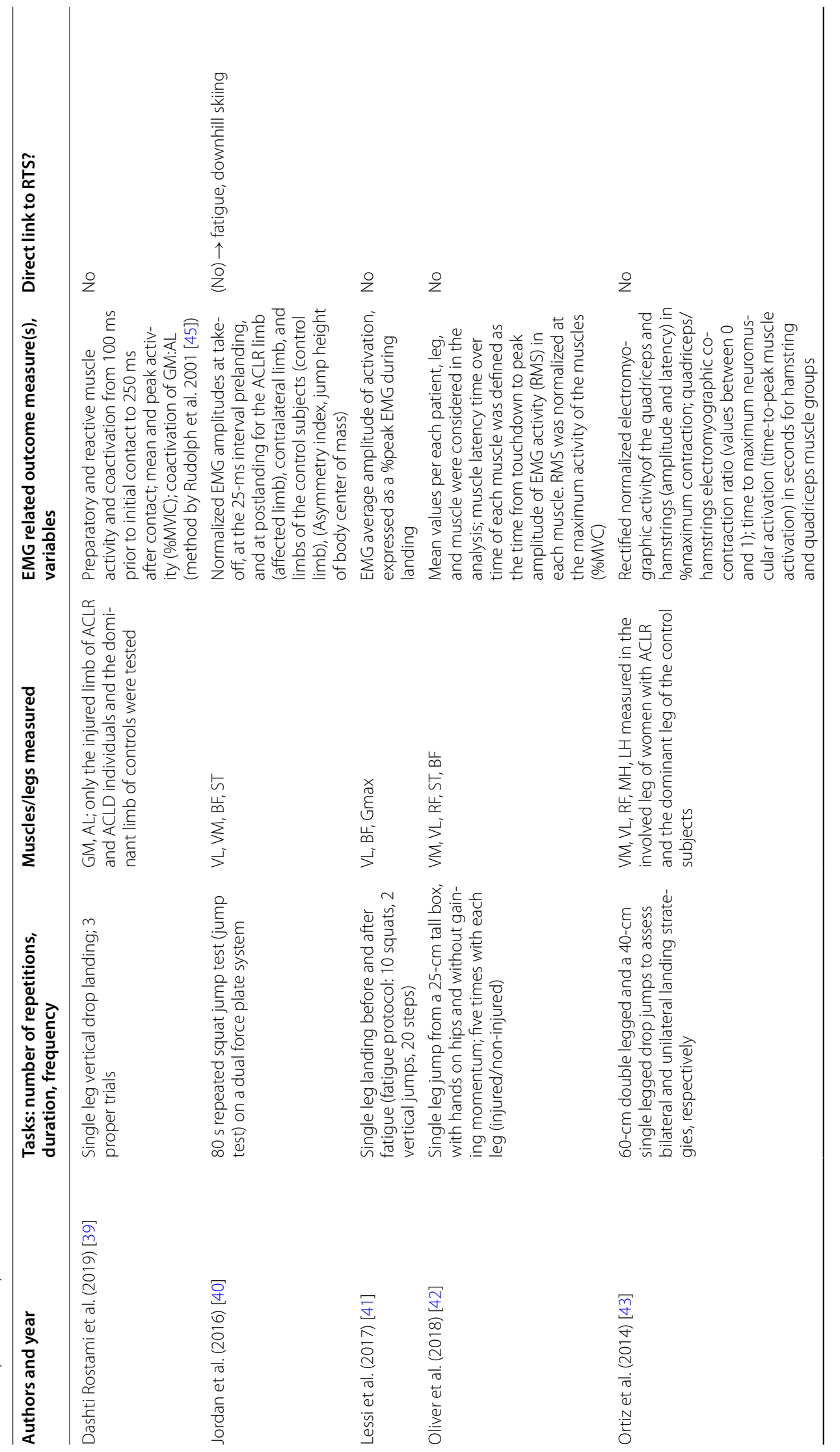




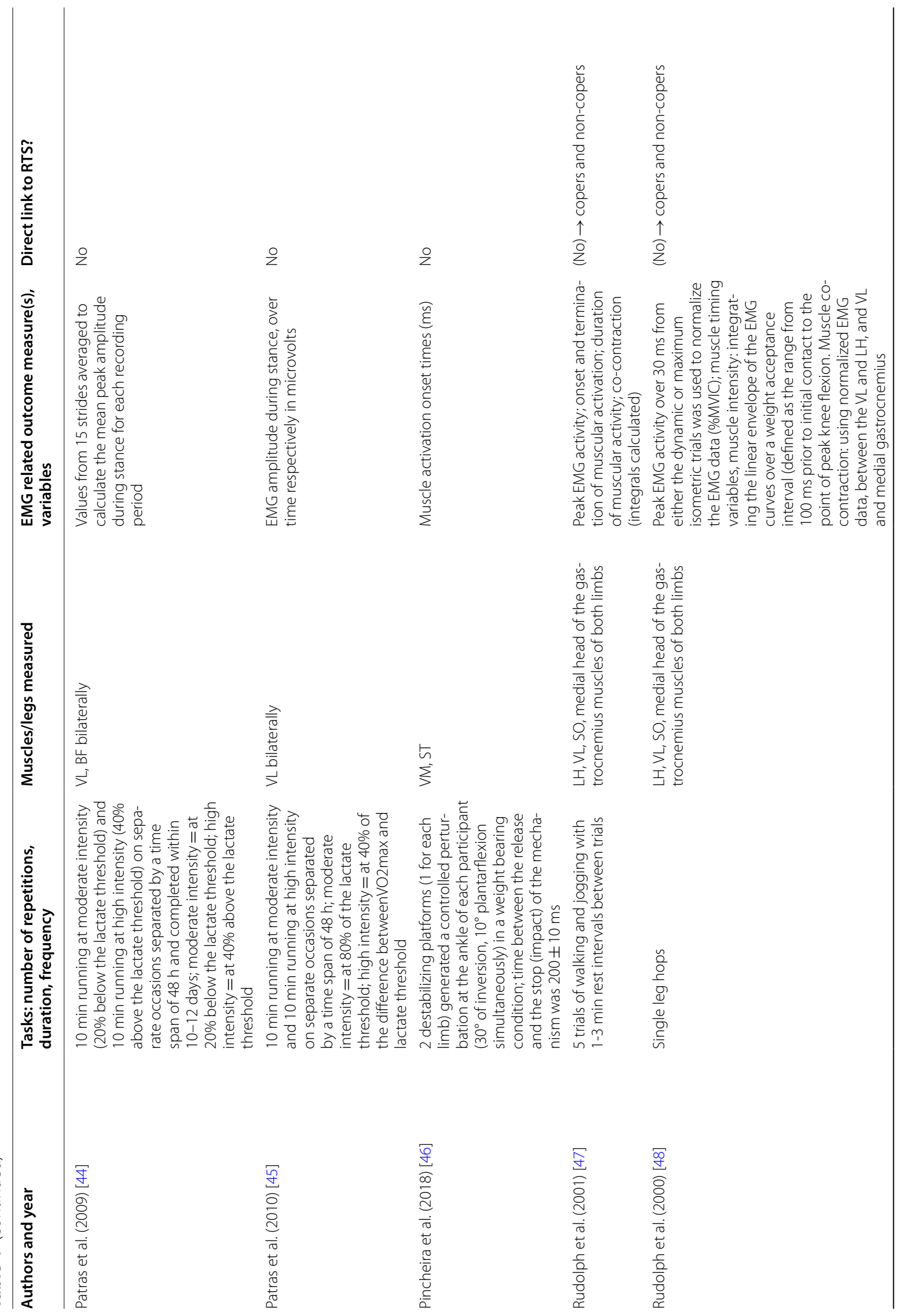




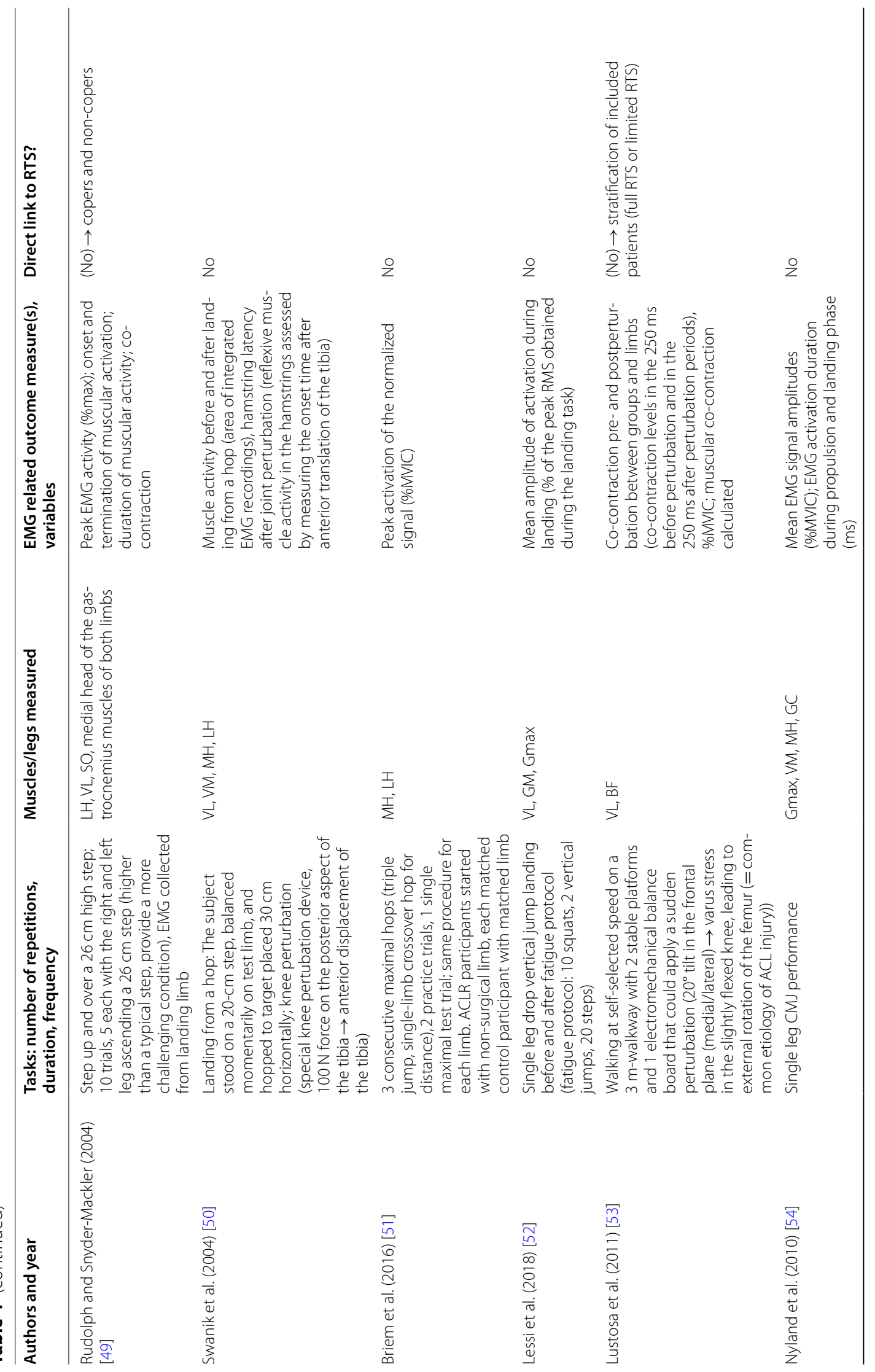




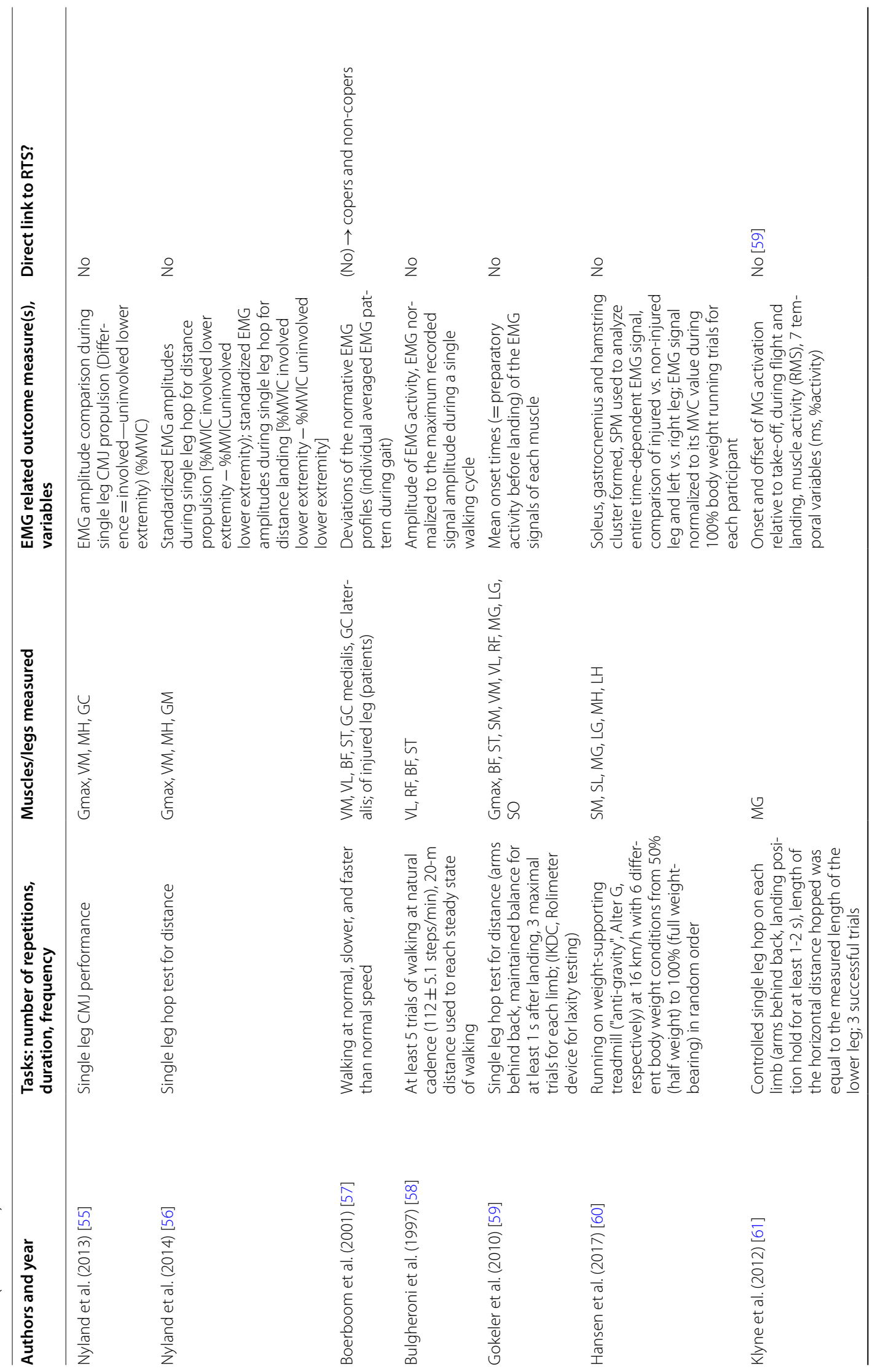




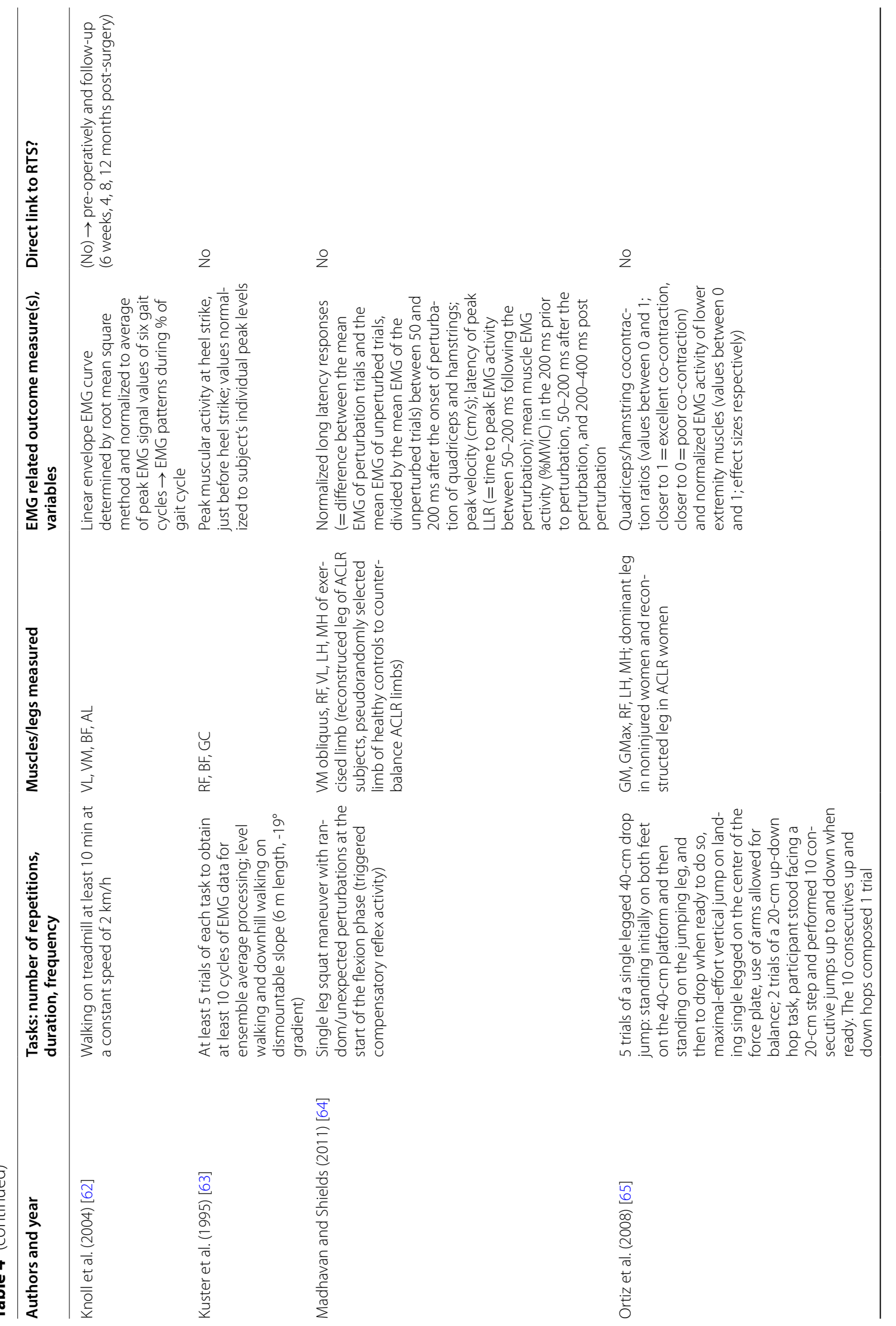




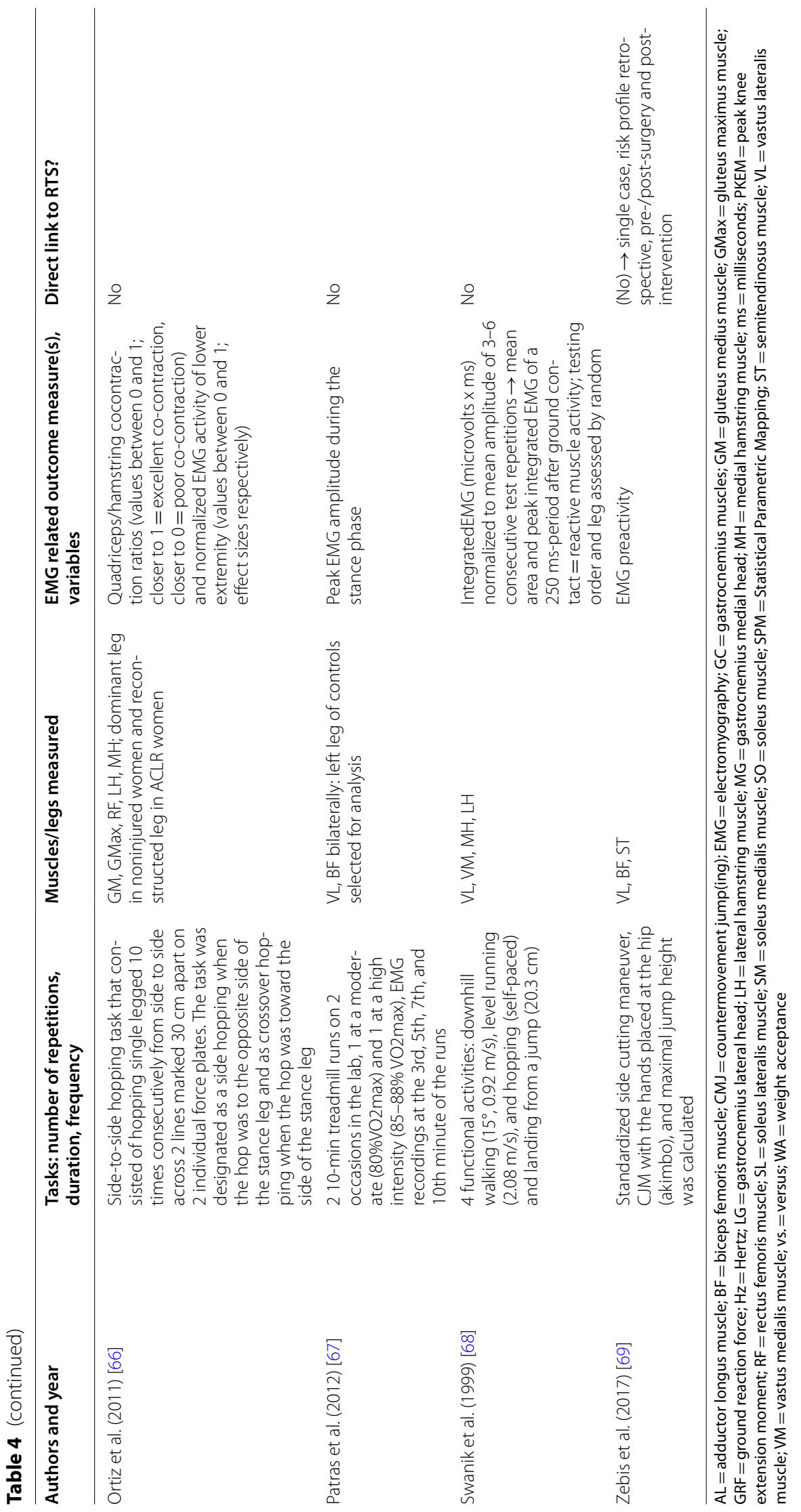


hamstrings or hamstrings curls [35] and squats [64]. At the other end of the scale, more complex, highly demanding, sport-specific tasks such as an instep soccer kick [38] or a sidecutting maneuver [69] were reported. Only few research groups used perturbation platforms to simulate injury mechanisms during walking [53] or squatting [46, 64], or applied devices to stress the ACL in the posterioranterior direction [50]. In addition, two studies even investigated the influence of fatigue on neuromuscular control $[41,52]$.

\section{Outcomes}

All included studies used surface EMG as method to assess neuromuscular control and provided EMG-related variables such as peak and mean amplitudes, timing and peak of muscle activity, preparatory and reactive muscle activity, on- and offset of muscular activation, coactivation/co-contraction ratios, or asymmetry index. The outcome variables were expressed as percentage of maximum voluntary (isometric) contraction (\%MVIC or $\% \mathrm{MVC}$ ) or reported in microvolts or milliseconds according to the variable chosen in amplitude or time domain.

\section{Decision for Return to Sports (RTS)}

None of the included studies used the surface EMG measurements to decide upon readiness for RTS (Table 4). However, the results from about a third of the studies (31.6\%, 12 studies) could provide useful information by the choice of the assessed groups such as copers versus non-copers [33, 34, 47-49,57], intervention and control group from the same team or level/league [35, $38,40]$, data from pre-injury/pre-surgery including postsurgical follow up [62,69] or participants with full RTS versus limited RTS [53]. In addition, two studies even investigated the influence of fatigue on neuromuscular control [41, 52].

More detailed information regarding EMG methods and procedures such as EMG type, detection, normalization, data processing and electrode placement can be found in Additional file "EMG methods and procedures of included studies" 3.

\section{Discussion}

The aim of this systematic review was to summarize the scientific literature regarding EMG-related assessments for neuromuscular control in patients with an ACL injury (either treated surgically or conservatively). The second aim was to analyze whether these assessments for neuromuscular control were used to decide upon readiness for RTS in these patients.

There were many factors present which could have an influence on neuromuscular control.

\section{Influence by type of comparison (intra- versus inter-subject)}

The use of the contralateral, non-injured leg in intrasubject comparison, without a "real" control group [42, 44] may lead to an overestimation of the physical performance in the ACL reconstructed or -injured leg. After ACLR, functional performance is often expressed with the LSI [70]. As the non-affected limb may also have deteriorated, the LSI may overestimate the right time for a safe RTS, and therefore, the risk for secondary injury may be higher [23]. In acutely injured ACL patients, intra-individual comparison showed bilateral consequences during stair ascent and indicates an alteration in the motor program ("pre-programmed activity") [71]. In addition, in case of a case-controlled study design, the subjects in the control group should be matched to the ACL participants regarding sex, age, body mass, height, activity level and leg dominance.

\section{Influence by level of activity and fatigue}

Some of the included studies used very challenging, sports-specific task to assess neuromuscular control, some even assessed neuromuscular control after fatiguing tasks. It is known that most of ACL tears are noncontact injuries happening at the end of a training session or a play [72]. Therefore, the closer the task to the sports and injury-risky situation, the safer the decision towards full RTS or even return to competition will be. However, assessing performance-based tests or movement quality may be more difficult to standardize, require more complex equipment and large amounts of space. But if only impairments will be tested, there will be a lack of information regarding an "athlete's capacity to cope with the physical and mental demands of playing sport" [73]. It is therefore recommended to search for a standardized assessment close to the injury mechanism.

\section{Influence by gender}

Not all included studies reported findings of mixed groups separately by gender. Some did not even state whether study participants were male or female. This could partly be explained by the date of publication as gender difference in ACL patients has not been in the focus of former ACL research. It is known that female athletes are more likely to sustain an ACL injury than men $[74,75]$; the increased risk is probably multifactorial [76]. Several studies indicate that hormonal factors play 
a role $[3,77]$ contributing to an increased laxity of ligaments in the first half of the menstrual cycle. However, biomechanical and neuromuscular aspects as indicators are discussed controversially in literature: Gender-specific neuromuscular adaptations and biomechanical landing techniques are considered being the most important ones to explain the increased risk of injury in women [78, 79]. The higher risk for females to suffer from an ACL injury can be explained by motion and loading of the knee joint during performance [74]. Female athletes typically perform movements in sports with a greater knee valgus angle than men. Therefore, the amount of stress on the ACL in these situations is higher caused by a high activation of the quadriceps despite limited knee and hip flexion, greater hip adduction and a large knee adduction moment $[80,81]$. The dominance of the quadriceps muscle in women could contribute to increased anterior tibial translation $[82,83]$ and was found in various activities such as jumps and cutting maneuvers [84-86]. Moreover, females typically land with an internally or externally rotated tibia [87], leading to an increased knee valgus stress due to greater and more laterally orientated ground reaction forces [83]. In contrast, other researchers did not find any gender-specific differences in the quadriceps-hamstrings ratio [88], not even in landing and cutting maneuvers [89]. A systematic review summarized biomechanical gender differences and stated that these were based on questionable clinical relevance [89]. In addition, strength-paired women and men showed no significant differences in neuromuscular activity [90].

\section{Influence by treatment}

The included studies reported different treatment options (ACLR with different graft types, conservative treatment). Depending on the classification of the participants in copers and non-copers, the results in neuromuscular control may differ from a population of ACLR participants. Therefore, all researchers who worked with copers and non-copers made intra- and inter-group comparisons without an ACLR group. A Cochrane review revealed low evidence for no difference in young, active adults after two and five years after the injury, assessed with patient-reported outcomes. However, many participants described as "non-copers" with unstable knee with conservative treatments remain symptomatic, and therefore, later opt for ACL surgery [91]. It has been described that persistent co-contraction and joint stiffening in these "non-copers" is likely to be due to an abnormal neuromuscular strategy failing to restore joint stability in these ACL deficient group [92]. Furthermore, the choice of graft would influence the neuromuscular control of measured muscles due to the morbidity of the harvesting site of the graft (e.g. hamstrings).

\section{EMG variables}

If the researchers mentioned the procedures for collecting EMG data, they referred to standardized applications and guidelines such as SENIAM (Surface Electromyography for the Non-Invasive Assessment of Muscles) [93]. The provided EMG-related variables were in accordance to the ones mentioned in a systematic review searching for knee muscle activity in ACL deficient patients and healthy controls during gait [14]. Current literature suggests greater co-contraction indices, increased joint stiffness and earlier muscle activation onset times as measures of neuromuscular function reflecting the incomplete restoration of normal joint stability $[19,92]$. Some of the included studies reported values of muscle onset activity in milliseconds and percentage of gait cycle as a systematic review did by summarizing and quantitatively analyzing muscle onset activity prior to landing in patients after ACL injury [20]. However, no cut-off values out of EMG-related variables were provided to determine an adequate level of neuromuscular control. Moreover, some of the researchers only provided integrated EMG values which would make it difficult to be compared to other studies using the respective units (milliseconds, millivolts) or widely used percentage values (\%MVIC, \%MVC).

\section{Return to sports (RTS)}

Regarding the determination of RTS after ACLR, there is some evidence for the use of functional performance tests, which had also been widely used in the included studies. Multiple functional performance measures-a battery including strength and hop tests, quality of movement and psychological tests [25] — might be more useful for the determination of RTS than a single performance measure. However, it is still unclear, which measures should be used to bring athletes safely back to RTS with a low risk of a second ACL injury [25]. Currently used RTS criteria or assessments, such as time, strength tests, hop tests, patient-reports, clinical examination, thigh circumference, ligamentous stability, range of motion, effusion and performance-based criteria, may be suboptimal at reducing the risk of a second ACL injury [73, 94]. Recovery of neuromuscular function was mentioned to be important because of the existing connection between the variables time since surgery and the risk for re-injury of the knee joint; but adequate assessment procedures to assess neuromuscular function are still a matter of debate [7]. In contrast, authors of an included study stated that "studies like ours that focus on the objective measurement of the change of the muscle latency time over time may allow patients to return to full activity and to sports earlier than the standard time of 6-12 months" [42]. 
However, this statement only based on one outcome measure and contrasts with current criterion- and timebased recommendations for RTS. Therefore, this recommendation seems to be rather dangerous.

\section{Limitations}

The sample size of all the studies was quite low, however, providing reasonable sample size calculations and depending on the variable investigated, the results were acceptable. Furthermore, the more restrictive the inclusion criteria for the participants, the more homogeneous the intervention and the control groups were, but the more challenging the recruitment process was, leading to smaller groups to be investigated.

The used assessment for the risk of bias, the Downs and Black checklist [31] in a modified form [29, 32] is designed for randomized and non-randomized controlled studies, however, the latter score lower in some items, get lower total scores and therefore a worse overall rating of the methodological quality. Despite this disadvantage, we decided to use the modified checklist as we could assess all studies with different designs included in this systematic review. However, the use of total scores and choice of cut-off values for low, medium and high risk of bias, respectively, were arbitrary and not based on literature.

\section{Conclusions}

\section{Implications for clinical practice}

This systematic review summarized assessments using EMG variables for neuromuscular control of the knee in patients suffering from an ACL injury (either treated surgically or conservatively). Despite 38 articles providing a wide range of EMG-related assessments, none was used to decide upon readiness towards a safe and successful RTS in patients after an ACL injury. So far, there is no diagnostic measure to assess neuromuscular control and therefore, clinicians should use a multimodal approach including assessments for active and passive knee stability under different sports-related conditions but be aware of not being able to evaluate neuromuscular control in depth without EMG-related assessments. Moreover, the widely used LSI may overestimate the physical performance of an ACL patient as the non-affected limb is likely to have deteriorated, too.

\section{Implications for further research}

Additional studies are needed to define readiness towards RTS by assessing neuromuscular control in adult ACL patients with EMG. Further research should aim at finding reliable and valid, EMG-related variables to be used as diagnostic tool for neuromuscular control.
Due to the heterogeneity in participants, interventions and outcomes used, future studies should aim at more homogenous patient groups, evaluate females and males separately, provide adequately matched healthy subjects (gender, height, weight, activity level etc.), control for confounding factors such as type of treatment, and use tasks close to the injury mechanism, as sport specific as possible, respectively. Moreover, it would be interesting to assess not only lower leg but pelvic and core muscles in addition. This would help to give insight in the complex field of ACL injuries and subsequent rehabilitation strategies, and therefore improve knowledge towards a safe RTS in these patients.

\section{Abbreviations \\ ACL: Anterior cruciate ligament; ACLR: Anterior cruciate ligament reconstruc- tion; EMG: Electromyography; LSI: Limb Symmetry Index; PEDro: Physiotherapy Evidence Database; PICOS: Participants-Intervention-Control-Outcome-Study design; PRISMA: Preferred Reporting of Items for Systematic reviews and Meta- Analyses; PROSPERO: International prospective register of systematic reviews; RTS: Return to sports; SENIAM: Surface Electromyography for the Non-Invasive Assessment of Muscles; TAS: Tegner Activity Score.}

\section{Supplementary Information}

The online version contains supplementary material available at https://doi. org/10.1186/s13102-021-00370-5.

Additional file 1 Search string for MEDLINE/PubMed.

Additional file 2 Methodological quality assessment.

Additional file 3 EMG methods and procedures of included studies.

\section{Acknowledgements}

We would like to thank to all the following people for their assistance and contribution regarding this work: the librarians of the Bern University of Applied Sciences (Department of Health Professions) and the University of Bern (Institute for Sports Science) for their support before and during the literature search, M. Akter for her help in extracting relevant key words and A. Busch for her critical review of the proposal and support to refine the search strategy.

\section{Authors' information}

AB, MPTSC, MSc in Physiotherapy, PT, is Deputy Head of the research group "Neuromuscular control" and Co-Leader of the Bachelor of Science in Physiotherapy at the Bern University of Applied Sciences, Department of Health Professions, Division of Physiotherapy in Bern (Switzerland). AB is well-trained in orthopedics, sports physiotherapy and active rehabilitation in different settings. She works as lecturer and researcher and does her doctoral studies at the University of Antwerp (Belgium) in the field of neuromuscular control and ACL injuries. IK, PhD, MSc in Physiotherapy, M.A. in Adult Education, PT, is Head of the research group "Pelvic Floor and Continence" and Co-Leader of the Bachelor of Science in Physiotherapy at the Bern University of Applied Sciences, Department of Health Professions, Division of Physiotherapy in Bern (Switzerland). She is well-trained in pelvic floor rehabilitation, EMG assessments and analyses with different methods. IB, PhD, PT, works as tenure track lecturer at the University of Antwerp, Faculty of Medicine and Health Sciences, Department of Rehabilitation Sciences and Physiotherapy in Wilrijk (Belgium) in the Bachelor and Master study programs. Her main interests regarding research are in the field of musculoskeletal diseases (lower extremity and lumbopelvic region), assessments in physiotherapy and pain management. $\mathrm{HB}$, Professor, PhD in Sport and Movement Science, is Head of Applied Research and Development and Head of the research group "Neuromuscular control" 
at the Bern University of Applied Sciences, Department of Health Professions, Division of Physiotherapy in Bern (Switzerland). His main interests in research are development of diagnostic and interventional assessments for musculoskeletal problems, influence of neuromuscular control by external (training/ exercise, therapy, aids) and internal factors (gender, age, etc.) and development of quality control measures in physiotherapy. DV, Professor, PhD, PT, is senior lecturer at the at the University of Antwerp, Faculty of Medicine and Health Sciences, Department of Rehabilitation Sciences and Physiotherapy in Wilrijk (Belgium). He has expertise in sports-related injuries, physical conditioning (muscle strength, motor control exercises), technology use in the field of physiotherapy, exercise therapy and rehabilitation in different pathologies. DV is first or co-author of several systematic reviews and meta-analysis.

\section{Authors' contributions}

AB participated in the design of the study, contributed to data collection/ reduction/analysis and interpretation of results and was the main contributor in writing the manuscript; IK contributed to data collection, reduction and analysis; IB and DV participated in the design of the study; HB participated in the design of the study and was an important contributor in writing the manuscript, contributed to data analysis and interpretation of results. All authors contributed to the manuscript writing, read, and approved the final version of the manuscript and agreed with the order of authors as listed.

\section{Funding}

The Bern University of Applied Sciences provided working hours for AB as grant for non-tenured staff, but was not involved in the design of the study, collection, analysis and interpretation of data, in writing the manuscript and in the decision to submit the article for publication.

\section{Availability of data and materials}

The datasets used and analyzed in the current study are available from the corresponding author on reasonable request.

\section{Declarations}

Ethics approval and consent to participate

Not applicable.

\section{Consent for publication}

Not applicable.

\section{Competing interests}

The authors declare that they have no competing interests.

\section{Author details}

'Department of Health Professions, Division of Physiotherapy, Bern University of Applied Sciences, Murtenstrasse 10, 3008 Bern, Switzerland. ${ }^{2}$ Department of Rehabilitation Sciences and Physiotherapy, Faculty of Medicine and Health Sciences, University of Antwerp, Campus Drie Eiken, Universiteitsplein 1, 2610 Wilrijk, Belgium.

Received: 3 February 2021 Accepted: 26 October 2021

Published online: 08 November 2021

\section{References}

1. Hootman JM, Dick R, Agel J. Epidemiology of collegiate injuries for 15 sports: summary and recommendations for injury prevention initiatives. J Athl Train. 2007:42(2):311-9.

2. Kaeding CC, Leger-St-Jean B, Magnussen RA. Epidemiology and diagnosis of anterior cruciate ligament injuries. Clin Sports Med. 2017;36(1):1-8.

3. Hewett TE, Zazulak BT, Myer GD. Effects of the menstrual cycle on anterior cruciate ligament injury risk: a systematic review. Am J Sports Med. 2007;35(4):659-68.

4. Friel NA, Chu CR. The role of ACL injury in the development of posttraumatic knee osteoarthritis. Clin Sports Med. 2013;32(1):1-12.

5. Ardern CL, Webster KE, Taylor NF, Feller JA. Return to sport following anterior cruciate ligament reconstruction surgery: a systematic review and meta-analysis of the state of play. Br J Sports Med. 2011;45(7):596-606.
6. Lai CCH, Feller JA, Webster KE. Fifteen-year audit of anterior cruciate ligament reconstructions in the australian football league from 1999 to 2013: return to play and subsequent ACL injury. Am J Sports Med. 2018;46(14):3353-60.

7. Grindem H, Snyder-Mackler L, Moksnes H, Engebretsen L, Risberg MA. Simple decision rules can reduce reinjury risk by $84 \%$ after $A C L$ reconstruction: the delaware-oslo ACL cohort study. Br J Sports Med. 2016;50(13):804-8.

8. Wright RW, Magnussen RA, Dunn WR, Spindler KP. Ipsilateral graft and contralateral ACL rupture at five years or more following ACL reconstruction: a systematic review. J Bone Joint Surg Am. 2011;93(12):1159-65.

9. Paterno MV, Rauh MJ, Schmitt LC, Ford KR, Hewett TE. Incidence of contralateral and ipsilateral anterior cruciate ligament (ACL) injury after primary ACL reconstruction and return to sport. Clin J Sport Med. 2012;22(2):116-21.

10. Paterno MV, Rauh MJ, Schmitt LC, Ford KR, Hewett TE. Incidence of second $A C L$ injuries 2 years after primary $A C L$ reconstruction and return to sport. Am J Sports Med. 2014;42(7):1567-73.

11. Akelman MR, Fadale PD, Hulstyn MJ, Shalvoy RM, Garcia A, Chin KE, et al. Effect of matching or overconstraining knee laxity during anterior cruciate ligament reconstruction on knee osteoarthritis and clinical outcomes: a randomized controlled trial with 84-month follow-up. Am J Sports Med. 2016;44(7):1660-70.

12. Group MK, Spindler KP, Huston LJ, Chagin KM, Kattan MW, Reinke EK, et al. Ten-year outcomes and risk factors after anterior cruciate ligament reconstruction: a MOON longitudinal prospective cohort study. Am J Sports Med. 2018;46(4):815-25.

13. Busch A, Blasimann A, Henle P, Baur H. Neuromuscular activity during stair descent in $\mathrm{ACL}$ reconstructed patients: a pilot study. Knee. 2019;26(2):310-6.

14. Shanbehzadeh S, Mohseni Bandpei MA, Ehsani F. Knee muscle activity during gait in patients with anterior cruciate ligament injury: a systematic review of electromyographic studies. Knee Surg Sports Traumatol Arthrosc. 2017;25(5):1432-42.

15. Mantashloo Z, Letafatkar A, Moradi M. Vertical ground reaction force and knee muscle activation asymmetries in patients with $\mathrm{ACL}$ reconstruction compared to healthy individuals. Knee Surg Sports Traumatol Arthrosc. 2020;28(6):2009-14.

16. Grooms DR, Page SJ, Nichols-Larsen DS, Chaudhari AM, White SE, Onate JA. Neuroplasticity associated with anterior cruciate ligament reconstruction. J Orthop Sports Phys Ther. 2017:47(3):180-9.

17. Zimny ML, Schutte M, Dabezies E. Mechanoreceptors in the human anterior cruciate ligament. Anat Rec. 1986;214(2):204-9.

18. Lanier AS, Knarr BA, Stergiou N, Snyder-Mackler L, Buchanan TS. ACL injury and reconstruction affect control of ground reaction forces produced during a novel task that simulates cutting movements. J Orthop Res. 2020;38(8):1746-52.

19. Nawasreh ZH, Marmon AR, Logerstedt D, Snyder-Mackler L. The effect of training on a compliant surface on muscle activation and co-contraction after anterior cruciate ligament injury. Int J Sports Phys Ther. 2019;14(4):3554-63.

20. Theisen D, Rada I, Brau A, Gette P, Seil R. Muscle activity onset prior to landing in patients after anterior cruciate ligament injury: a systematic review and meta-analysis. PLoS ONE. 2016;11(5):e0155277.

21. Ford KR, van den Bogert J, Myer GD, Shapiro R, Hewett TE. The effects of age and skill level on knee musculature co-contraction during functional activities: a systematic review. Br J Sports Med. 2008;42(7):561-6.

22. Tsai LC, McLean S, Colletti PM, Powers CM. Greater muscle co-contraction results in increased tibiofemoral compressive forces in females who have undergone anterior cruciate ligament reconstruction. J Orthop Res. 2012;30(12):2007-14.

23. Wellsandt E, Failla MJ, Snyder-Mackler L. Limb symmetry indexes can overestimate knee function after anterior cruciate ligament injury. J Orthop Sports Phys Ther. 2017;47(5):334-8.

24. Webster KE, Feller JA. A research update on the state of play for return to sport after anterior cruciate ligament reconstruction. J Orthop Traumatol. 2019;20(1):10.

25. van Melick N, van Cingel RE, Brooijmans F, Neeter C, van Tienen T, Hullegie W, et al. Evidence-based clinical practice update: practice guidelines for anterior cruciate ligament rehabilitation based on a systematic review and multidisciplinary consensus. Br J Sports Med. 2016;50(24):1506-15. 
26. Bruhn S, Leukel C, Gollhofer A. Differential effects of stimulus characteristics during knee joint perturbation on hamstring and quadriceps reflex responses. Hum Move Sci. 2011;30(6):1079-91.

27. Moher D, Liberati A, Tetzlaff J, Altman DG, Group P. Preferred reporting items for systematic reviews and meta-analyses: the PRISMA statement. PLoS Med. 2009;6(7):e1000097.

28. Higgins JPT, Green S. Cochrane handbook for systematic reviews of interventions. Chichester: Wiley; 2008.

29. Ramsey CA, Lamb P, Kaur M, Baxter GD, Ribeiro DC. How are running shoes assessed? A systematic review of characteristics and measurement tools used to describe running footwear. J Sports Sci. 2019;37(14):1617-29.

30. Ouzzani M, Hammady H, Fedorowicz Z, Elmagarmid A. Rayyan-a web and mobile app for systematic reviews. Syst Rev. 2016;5(1):210.

31. Downs SH, Black N. The feasibility of creating a checklist for the assessment of the methodological quality both of randomised and nonrandomised studies of health care interventions. J Epidemiol Community Health. 1998;52(6):377-84.

32. Pairot-de-Fontenay B, Willy RW, Elias ARC, Mizner RL, Dube MO, Roy JS. Running biomechanics in individuals with anterior cruciate ligament reconstruction: a systematic review. Sports Med (Auckland, NZ). 2019:49(9):1411-24.

33. Alkjaer T, Simonsen EB, Jorgensen U, Dyhre-Poulsen P. Evaluation of the walking pattern in two types of patients with anterior cruciate ligament deficiency: copers and non-copers. Eur J Appl Physiol. 2003;89(3):301-8.

34. Alkjaer T, Simonsen EB, Peter Magnusson SP, Aagaard H, Dyhre-Poulsen P. Differences in the movement pattern of a forward lunge in two types of anterior cruciate ligament deficient patients: copers and non-copers. Clin Biomech (Bristol, Avon). 2002;17(8):586-93.

35. Arnason SM, Birnir B, Guomundsson TE, Guonason G, Briem K. Medial hamstring muscle activation patterns are affected 1-6 years after ACL reconstruction using hamstring autograft. Knee Surg Sports Traumatol Arthrosc. 2014;22(5):1024-9.

36. Bryant AL, Newton RU, Steele J. Successful feed-forward strategies following ACL injury and reconstruction. J Electromyogr Kinesiol. 2009;19(5):988-97.

37. Burland JP, Lepley AS, Frechette L, Lepley LK. Protracted alterations in muscle activation strategies and knee mechanics in patients after anterior cruciate ligament reconstruction. Knee Surg Sports Traumatol Arthrosc. 2020;28(12):3766-72

38. Cordeiro N, Cortes N, Fernandes O, Diniz A, Pezarat-Correia P. Dynamic knee stability and ballistic knee movement after $\mathrm{ACL}$ reconstruction: an application on instep soccer kick. Knee Surg Sports Traumatol Arthrosc: Off J ESSKA. 2015;23(4):1100-6.

39. Dashti Rostami K, Naderi A, Thomas A. Hip Abductor and adductor muscles activity patterns during landing after anterior cruciate ligament injury. J Sport Rehabil. 2019;28:871-6.

40. Jordan MJ, Aagaard P, Herzog W. Asymmetry and thigh muscle coactivity in fatigued anterior cruciate ligament-reconstructed elite skiers. Med Sci Sports Exer. 2017;49(1):11-20.

41. Lessi GC, Serrao FV. Effects of fatigue on lower limb, pelvis and trunk kinematics and lower limb muscle activity during single-leg landing after anterior cruciate ligament reconstruction. Knee Surg Sports Traumatol Arthrosc. 2017;25(8):2550-8.

42. Oliver G, Portabella F, Hernandez JA. A comparative study of the neuromuscular response during a dynamic activity after anterior cruciate ligament reconstruction. Eur J Orthop Surg Traumatol: Orthop Traumatol. 2019:29:633-8.

43. Ortiz A, Capo-Lugo CE, Venegas-Rios HL. Biomechanical deficiencies in women with semitendinosus-gracilis anterior cruciate ligament reconstruction during drop jumps. PM \& R: I Injury Funct Rehab. 2014;6(12):1097-106.

44. Patras K, Ziogas G, Ristanis S, Tsepis E, Stergiou N, Georgoulis AD. High intensity running results in an impaired neuromuscular response in ACL reconstructed individuals. Knee Surg Sports Traumatol Arthrosc. 2009:17(8):977-84.

45. Patras K, Ziogas G, Ristanis S, Tsepis E, Stergiou N, Georgoulis AD. ACL reconstructed patients with a BPTB graft present an impaired vastus lateralis neuromuscular response during high intensity running. J Sci Med Sport. 2010;13(6):573-7.
46. Pincheira PA, Silvestre R, Armijo-Olivo S, Guzman-Venegas R. Ankle perturbation generates bilateral alteration of knee muscle onset times after unilateral anterior cruciate ligament reconstruction. PeerJ. 2018;6:19.

47. Rudolph KS, Axe MJ, Buchanan TS, Scholz JP, Snyder-Mackler L. Dynamic stability in the anterior cruciate ligament deficient knee. Knee Surg Sports Traumatol Arthrosc. 2001;9(2):62-71.

48. Rudolph KS, Axe MJ, Snyder-Mackler L. Dynamic stability after ACL injury: who can hop? Knee Surg Sports Traumatol Arthrosc: Off J ESSKA. 2000;8(5):262-9.

49. Rudolph KS, Snyder-Mackler L. Effect of dynamic stability on a step task in ACL deficient individuals. J Electromyogr Kinesiol. 2004;14(5):565-75.

50. Swanik CB, Lephart SM, Swanik KA, Stone DA, Fu FH. Neuromuscular dynamic restraint in women with anterior cruciate ligament injuries. Clin Orthop Related Res. 2004;425:189-99.

51. Briem K, Ragnarsdottir AM, Arnason SI, Sveinsson T. Altered medial versus lateral hamstring muscle activity during hop testing in female athletes 1-6 years after anterior cruciate ligament reconstruction. Knee Surg Sports Traumatol Arthrosc: Off J ESSKA. 2016;24(1):12-7.

52. Lessi GC, Silva RS, Serrao FV. Comparison of the effects of fatigue on kinematics and muscle activation between men and women after anterior cruciate ligament reconstruction. Phys Ther Sport. 2018;31:29-34.

53. Lustosa LP, Ocarino JM, de Andrade MA, Pertence AE, Bittencourt NF, Fonseca ST. Muscle co-contraction after anterior cruciate ligament reconstruction: influence of functional level. J Electromyogr Kinesiol: Off J Int Soc Electrophysiol Kinesiol. 2011;21(6):1050-5.

54. Nyland J, Klein S, Caborn DNM. Lower extremity compensatory neuromuscular and biomechanical adaptations 2 to 11 years after anterior cruciate ligament reconstruction. Arthrosc-J Arthroscop Relat Surg. 2010:26(9):1212-25

55. Nyland J, Mauser N, Caborn DNM. Sports involvement following ACL reconstruction is related to lower extremity neuromuscular adaptations, subjective knee function and health locus of control. Knee Surg Sports Traumatol Arthrosc. 2013;21(9):2019-28.

56. Nyland J, Wera J, Klein S, Caborn DN. Lower extremity neuromuscular compensations during instrumented single leg hop testing $2-10$ years following ACL reconstruction. Knee. 2014;21(6):1191-7.

57. Boerboom AL, Hof AL, Halbertsma JP, van Raaij JJ, Schenk W, Diercks RL, et al. Atypical hamstrings electromyographic activity as a compensatory mechanism in anterior cruciate ligament deficiency. Knee Surg Sports Traumatol Arthrosc. 2001;9(4):211-6.

58. Bulgheroni P, Bulgheroni MV, Andrini L, Guffanti P, Giughello A. Gait patterns after anterior cruciate ligament reconstruction. Knee Surg Sports Traumatol Arthrosc. 1997;5(1):14-21.

59. Gokeler A, Hof AL, Arnold MP, Dijkstra PU, Postema K, Otten E. Abnormal landing strategies after ACL reconstruction. Scand J Med Sci Sports. 2010:20(1):e12-9.

60. Hansen C, Einarson E, Thomson A, Whiteley R, Witvrouw E. Hamstring and calf muscle activation as a function of bodyweight support during treadmill running in ACL reconstructed athletes. Gait Posture. 2017;58:154-8.

61. Klyne DM, Keays SL, Bullock-Saxton JE, Newcombe PA. The effect of anterior cruciate ligament rupture on the timing and amplitude of gastrocnemius muscle activation: a study of alterations in EMG measures and their relationship to knee joint stability. J Electromyogr Kinesiol: Off J Int Soc Electrophysiol Kinesiol. 2012;22(3):446-55.

62. Knoll Z, Kocsis L, Kiss RM. Gait patterns before and after anterior cruciate ligament reconstruction. Knee Surg Sports Traumatol Arthrosc. 2004;12(1):7-14.

63. Kuster M, Blatter G, Hauswirth L, Neuer W, Wood GA. The anterior cruciate ligament, an important structure of the knee joint. Praxis (Bern 1994). 1995:84(5):134-9.

64. Madhavan S, Shields RK. Neuromuscular responses in individuals with anterior cruciate ligament repair. Clin Neurophysiol. 2010;122(5):997-1004.

65. Ortiz A, Olson S, Libby CL, Trudelle-Jackson E, Kwon YH, Etnyre B, et al. Landing mechanics between noninjured women and women with anterior cruciate ligament reconstruction during 2 jump tasks. Am J Sports Med. 2008;36(1):149-57.

66. Ortiz A, Olson S, Trudelle-Jackson E, Rosario M, Venegas HL. Landing mechanics during side hopping and crossover hopping maneuvers in noninjured women and women with anterior cruciate ligament reconstruction. PM \& R: J Injury Funct Rehab. 2011;3(1):13-20. 
67. Patras K, Zampeli F, Ristanis S, Tsepis E, Ziogas G, Stergiou N, et al. Hamstring-dominant strategy of the bone-patellar tendon-bone graft anterior cruciate ligament-reconstructed leg versus quadriceps-dominant strategy of the contralateral intact leg during high-intensity exercise in male athletes. Arthroscopy. 2012;28(9):1262-70.

68. Swanik CB, Lephart SM, Giraldo JL, Demont RG, Fu FH. Reactive muscle firing of anterior cruciate ligament-injured females during functional activities. J Athl Train. 1999;34(2):121-9.

69. Zebis MK, Andersen CH, Bencke J, Orntoft C, Linnebjerg C, Holmich P, et al. Neuromuscular coordination deficit persists 12 months after $A C L$ reconstruction but can be modulated by 6 weeks of kettlebell training: a case study in women's elite soccer. Case Rep Orthop. 2017;2017:4269575.

70. Engelen-van Melick N, van Cingel RE, Tijssen MP, Nijhuis-van der Sanden MW. Assessment of functional performance after anterior cruciate ligament reconstruction: a systematic review of measurement procedures. Knee Surg Sports Traumatol Arthrosc. 2013;21(4):869-79.

71. Busch A, Helne P, Boesch L, Blasimann A, Baur H. Neuromuscular control in patients with acute $\mathrm{ACL}$ injury during stair ascent-a pilot study. Sports Orthop Traumatol. 2019;35(2):158-65.

72. Price RJ, Hawkins RD, Hulse MA, Hodson A. The football association medical research programme: an audit of injuries in academy youth football. Br J Sports Med. 2004;38(4):466-71.

73. Burgi CR, Peters S, Ardern CL, Magill JR, Gomez CD, Sylvain J, et al. Which criteria are used to clear patients to return to sport after primary $\mathrm{ACL}$ reconstruction? A scoping review. Br J Sports Med. 2019;53(18):1154-61.

74. Hewett TE, Myer GD, Ford KR, Heidt RS Jr, Colosimo AJ, McLean SG, et al. Biomechanical measures of neuromuscular control and valgus loading of the knee predict anterior cruciate ligament injury risk in female athletes: a prospective study. Am J Sports Med. 2005;33(4):492-501.

75. Montalvo AM, Schneider DK, Yut L, Webster KE, Beynnon B, Kocher $M S$, et al. "What's my risk of sustaining an $A C L$ injury while playing sports?" A systematic review with meta-analysis. Br I Sports Med. 2019;53(16):1003-12.

76. Balachandar V, Marciniak JL, Wall O, Balachandar C. Effects of the menstrual cycle on lower-limb biomechanics, neuromuscular control, and anterior cruciate ligament injury risk: a systematic review. Muscles Ligaments Tendons J. 2017;7(1):136-46.

77. Belanger L, Burt D, Callaghan J, Clifton S, Gleberzon BJ. Anterior cruciate ligament laxity related to the menstrual cycle: an updated systematic review of the literature. J Can Chiropr Assoc. 2013;57(1):76-86.

78. Voskanian N. ACL Injury prevention in female athletes: review of the literature and practical considerations in implementing an $\mathrm{ACL}$ prevention program. Curr Rev Musculoskelet Med. 2013;6(2):158-63.

79. Wojtys EM, Wylie BB, Huston LJ. The effects of muscle fatigue on neuromuscular function and anterior tibial translation in healthy knees. Am J Sports Med. 1996;24(5):615-21.

80. Pappas E, Carpes FP. Lower extremity kinematic asymmetry in male and female athletes performing jump-landing tasks. J Sci Med Sport. 2012;15(1):87-92.

81. Pollard CD, Sigward SM, Powers CM. Limited hip and knee flexion during landing is associated with increased frontal plane knee motion and moments. Clin Biomech (Bristol, Avon). 2010;25(2):142-6.
82. Hanson AM, Padua DA, Troy Blackburn J, Prentice WE, Hirth CJ. Muscle activation during side-step cutting maneuvers in male and female soccer athletes. J Athl Train. 2008;43(2):133-43.

83. Sigward SM, Powers CM. Loading characteristics of females exhibiting excessive valgus moments during cutting. Clin Biomech (Bristol, Avon). 2007;22(7):827-33.

84. Malinzak RA, Colby SM, Kirkendall DT, Yu B, Garrett WE. A comparison of knee joint motion patterns between men and women in selected athletic tasks. Clin Biomech (Bristol, Avon). 2001;16(5):438-45.

85. Myer GD, Ford KR, Hewett TE. The effects of gender on quadriceps muscle activation strategies during a maneuver that mimics a high ACL injury risk position. J Electromyogr Kinesiol: Off J Int Soc Electrophysiol Kinesiol. 2005;15(2):181-9.

86. Padua DA, Carcia CR, Arnold BL, Granata KP. Gender differences in leg stiffness and stiffness recruitment strategy during two-legged hopping. J Mot Behav. 2005;37(2):111-25.

87. Nagano Y, Ida H, Akai M, Fukubayashi T. Gender differences in knee kinematics and muscle activity during single limb drop landing. Knee. 2007;14(3):218-23.

88. Hannah R, Folland JP, Smith SL, Minshull C. Explosive hamstrings-toquadriceps force ratio of males versus females. Eur J Appl Physiol. 2015;115(4):837-47.

89. Benjaminse A, Gokeler A, Fleisig GS, Sell TC, Otten B. What is the true evidence for gender-related differences during plant and cut maneuvers? A systematic review. Knee Surg Sports Traumatol Arthrosc. 2011;19(1):42-54

90. Nimphius S, McBride JM, Rice PE, Goodman-Capps CL, Capps CR. Comparison of quadriceps and hamstring muscle activity during an isometric squat between strength-matched men and women. J Sports Sci Med. 2019;18(1):101-8.

91. Monk AP, Davies LJ, Hopewell S, Harris K, Beard DJ, Price AJ. Surgical versus conservative interventions for treating anterior cruciate ligament injuries. Cochrane Database Syst Rev. 2016;4:CD011166.

92. Behnke AL, Parola LR, Karamchedu NP, Badger GJ, Fleming BC, Beveridge $J E$. Neuromuscular function in anterior cruciate ligament reconstructed patients at long-term follow-up. Clin Biomech (Bristol, Avon). 2021:81:105231.

93. Hermens HJ, Freriks B, Disselhorst-Klug C, Rau G. Development of recommendations for SEMG sensors and sensor placement procedures. J Electromyogr Kinesiol: Off J Int Soc Electrophysiol Kinesiol. 2000;10(5):361-74.

94. Losciale JM, Zdeb RM, Ledbetter L, Reiman MP, Sell TC. The association between passing return-to-sport criteria and second anterior cruciate ligament injury risk: a systematic review with meta-analysis. J Orthop Sports Phys Ther. 2019;49(2):43-54.

\section{Publisher's Note}

Springer Nature remains neutral with regard to jurisdictional claims in published maps and institutional affiliations.

Ready to submit your research? Choose BMC and benefit from:

- fast, convenient online submission

- thorough peer review by experienced researchers in your field

- rapid publication on acceptance

- support for research data, including large and complex data types

- gold Open Access which fosters wider collaboration and increased citations

- maximum visibility for your research: over $100 \mathrm{M}$ website views per year

At BMC, research is always in progress.

Learn more biomedcentral.com/submissions 